\title{
Fourmis du Congo
}

et d'autres provenances

récoltées

par MM. Hermann Kohl, Luja, Mayné, etc.

PAR

\section{Aug. FOREL}

Avec 7 figures dans le texte.

Je laisse de còté les formes déja fort connues ou n'offrant pas d'intérèt.

\section{$\mathrm{I}^{\text {re }}$ Sous-famille Ponerinae Lep.}

Ponera coeca Sant.

ㅇ. St-Gabriel, Congo (KoHL).

Ponera ergatandria For. r. Petri n. st.

ఢ. Longueur: $2^{\mathrm{mm}}, 9$. Ressemble à la r. cognata Sant., mais elle présente au milieu de l'épistome, devant, un sillon longitudinal qui rappelle celui de la sulcatinasis Sant. Du reste, beaucoup plus grande et avec l'écaille moins épaisse et plus haute. Les yeux sont plus grands que chez la r. cognata, comprenant 5 à 6 facettes plates. La tête est plus large, surtout derrière, aussi plus échancrée et un peu plus courte. La couleur

Rev. Suisse de Zool. T. 24. 1916. 
est d'un brun roussâtre ou d'un roux brunâtre avec les antennes et les mandibules d'un roux jaunàtre. La pubescence est plus forte que chez la r. cognata, mais moins forte que chez la sulcatinasis. Peut-être ferait-on mieux de rapporter cette forme comme race à la sulcatinasis.

St-Gabriel, Congo (КонL).

Plectroctena minor Em.

ґ. ․ St-Gabriel, Congo (KoHL).

\section{Platythyrea Conradti Em.}

$\zeta o^{\top}$. S'-Gabriel, Congo (КонL). Habite des arbres creux et leurs branches. Les trous de sortie sont recouverts de fines matières végétales, dans lesquelles on trouve des Fourmis parasites. Piqûre fort douloureuse.

Platythyrea modesta Em.

†. S'-Gabriel, Congo (KонL).

Pachycondyla (Bothroponera) Sveni n. sp.

Longueur: $9^{m m}-10^{m m}, 5$. Bien plus grande que sa parente la gabonensis André. Mandibules lisses, luisantes, avec de gros points épars et quelques rides vers l'extrémité, armées de quatre dents usées; le bord terminal peu distinct du bord interne. Tète rectangulaire, un peu plus longue que large, aussi large devant que derrière, à bord postérieur légèrement concave et à còtés faiblement convexes. Epistome sillonné au milieu, mais sans dents devant (avec 2 dents chez la gabonensis). L'épistome est avancé devant; son bord antérieur est convexe. Yeux assez grands, convexes, situés distinctement en avant du milieu. Le scape dépasse un peu le bord postérieur de la tête. Articles 2 à 6 du funicule plus longs qu'épais; articles 7 à 10 aussi épais que longs. Suture promésonotale distincte; suture mésoépinotale obsolète. Pronotum 2 fois plus large que long, un peu subépaulé. Epinotum armé de 2 épines divergentes, obtuses à l'extrémité, aussi longues que l'intervalle de leurs bases et plus longues que cette dernière, dirigées en haut et un peu en arrière. Face déclive 
avec une légère carène longitudinale. Ecaille semblable à celle de la gabonensis, mais les épines sont bien plus longues (comparées avec la figure de la v. striatidens Sant.). Les 2 épines latérales sont presque aussi longues que la hauteur de l'écaille. Les 3 épines médianes sont étroites et très longues, mais plus courtes que les latérales; la médiane environ 5 fois plus longue que la largeur de sa base. Abdomen à peine rétréci derrière son premier segment.

Entièrement male ou subopaque; thorax et tète plutòt subopaques. Tête assez grossièrement réticulée avec un peu de fine sculpture au fond des réticulations, et, entre deux, avec des rides longitudinales, surtout sur le front. Thorax grossièrement réticulé et assez finement et irrégulièrement sculpté au fond, avec des rides transversales grossières sur le pronotum; écaille plus luisante, avec des mailles réliculées, allongées, assez grossières. Sauf sa face antérieure, l'abdomen est mat, assez densément et finement strié en long, avec des réticulations allongées entre les stries et des points piligères, saillants et épars. Pilosité dressée abondante, assez épaisse, mais pointue au bout, plutòt courte, d'un brun roussâtre sur le corps, rousse sur les membres. Pubescence assez abondante et formant un léger duvet sur les membres, qui sont subopaques et densément ponctués. La pubescence est éparse sur le corps. Noire. Mandibules, scape, pattes, lobes des arètes frontales, devant des joues et de l'épistome, bord postérieur des segments abdominaux et bord antérieur des articles du funicule roussâtres. Cuisses et reste des funicules bruns.

Congo (Kонl). Reçue par M. Wasmann. Cette espèce diffère de la gabonensis par les longues épines de l'écaille et de l'épinotum, par sa grande taille, par le manque de dents à l'épistome, ainsi que par sa sculpture, etc.

Leptogenys Stuhlmanni Mayr r. camerunensis Stitz v. angusticeps n. var.

భ. Longueur: $9^{\mathrm{mm}}, 7$. Diffère du type de camerunensis par sa tête plus étroite et sa couleur bien plus foncée, brune, avec les 
A. FOREL

pattes, les antennes, les mandibules et les extrémités des segments abdominaux seules roussâtres. Chez le type de la camerunensis, qui est entièrement roussâtre, la tête est aussi large devant que sa longueur, tandis qu'elle est bien plus longue chez la v. angusticeps, avec les côtés distinctement plus convexes.

St-Gabriel, Congo (Конц), un seul exemplaire. Je crois devoir faire une race distincte de la camerunensis Stitz.

Anochetus Traegaordhi Mayr.

广. Congo (KонL).

Anochetus Bequaerti For.

†. Congo (KонL). $\subsetneq$ (non encore décrite). Longueur : $7^{\mathrm{mm}}, 5$. Tout à fait semblable à l'ouvrière; les mêmes dents obtuses et courtes à l'épinotum. Mésonotum subopaque, densément strié en long. Ailes un peu enfumées. Couleur du corps un peu plus claire que chez le type, d'un brun roussâtre. Pronotum avec quelques rides transversales.

Phyracaces nkomoensis $\mathrm{n}$. sp.

ఢ. Longueur: $4^{\mathrm{mm}}, 2-^{\prime m m}, 6$. Fort rapproché de Cooperi Arnold et de Foreli Sant. Mandibules lisses et luisantes avec de gros points épars. Arêtes latérales des joues, courtes, luisantes, mais élevées. Yeux au milieu, convexes, occupant près du tiers du còté de la tête. Articles 2 à 7 du funicule 2 fois plus épais que longs; les 3 derniers articles forment uné massue.

Le scape atteint le tiers postérieur de la tête. Celle-ci plus longue que large (d'1/5 à peine), avec les côtés convexes, mais aplaris derrière et bordés en dessous ; le bord postérieur largement échancré et un peu plus large que le bord antérieur. Thorax fortement convexe d'avant en arrière, plus faiblement de droite à gauche, sans sutures, mais avec un bord aigu, tout le long des côtés et entre la face basale et la face déclive de l'épinolum; ce dernier bord est interrompu au milieu. Le dos du thorax n'est pas tout à fait 2 fois plus long que large. Face déclive de l'épinotum très abrupte, plane, bordée latéralement, ayant vers son 
milieu deux petites carènes longitudinales; elle est séparée de la face basale par deux très petites dents. Pétiole rectangulaire, $1^{1 / 3}$ fois plus large que long, concave devant, convexe de còté et derrière, bordé devant et de còté, distinctement convexe dessus, ayant à ses angles postérieurs deux petites dents triangulaires, plus grandes que relles de l'épinotum. Sa face antérieure, un peu concave, est verticalement tronquée et bordée aussi de bas en haut. En bas, dessous, le pétiole a un lobe triangulaire. Postpétiole $1^{2 / 3}$ fois plus long que le pétiole et $1 \frac{1}{4}$ fois plus long que large. Distinctement plus large que le pétiole, avec les còtés un peu convexes, surtout assez fortement convexe dessus et dessous, ayant bien plus l'aspect d'un segment abdominal étranglé derrière que chez le Cooperi, il est néanmoins bordé et concave devant, ayant dessous, devant, une dent obtuse. Abdomen proprement dit largement articulé au postpétiole avec lequel il forme du reste un fort élranglement. Pattes moyennes ; les cuisses ont un lobe articulaire fort grand à leur extrémité postérieure, avant laquelle il est d'ailleurs aminci.

Luisant, avec une forte ponctuation profonde, égale et assez dense, sur tout le corps, bien plus fine et plus espacée, et bien plus superficielle sur les membres. Pilosité dressée, roussâtre, irrégulière, assez longue, éparse sur le corps et sur les membres, plus abondante sur l'aplatissement postérieur subbordé du pygidium. Pubescence éparse, peu apparente partout. Noir ; pattes et antennes brunes; tarses, aplatissement du pygidium, extrémité des mandibules et massue des antennes roussâtres.

S'-Gabriel, Congo (KoнL). Diffère de Cooperi et de Braunsi par la forme du postpéliole; de Foreli par sa sculpture tout autre. Le thorax est bien plus franchement bordé que chez Cooperi. La tête est plus large derrière et plus échancrée que chez Cooperi r. congolensis.

Phyracaces Cooperi Arnold r. congolensis n. st.

$\Varangle$. Longueur: $4^{\mathrm{mm}}, 2$. Diffère du type d'Arnold par sa tête bien plus étroite derrière et plutòt plus large devant, puis surtout par sa ponctuation infiniment plus fine, aussi fine sur tout 
le corps que sur l'abdomen, plus espacée que chez nkomoensis. Les antennes forment une massue bien moins distincte que chez ce dernier. Les angles de l'épinotum et du pétiole sont à peine dentiformes. Le postpétiole, bien plus nodiforme que chez nkomoensis, bien moins largement articulé à l'abdomen proprement dit, est distinctement plus large que long, tout à fait rectangulaire, bordé de còté. Du reste, comme le type.

St-Gabriel, Congo (КонL). D'après Arnold le Cooperi diffère de Braunsi par sa taille plus grande, ses yeux plus petits, ses scapes plus longs et sa ponctuation plus forte.

\section{$2^{\text {me }}$ Sous-famille Dorylinae Leach.}

Dorylus (Anomma) funereus $\mathrm{Em}$.

$\sigma^{x}$ Congo (Конц).

Dorylus (Anomma) Wilwerthi Em.

$\sigma^{\top}$ Congo (KонL).

Dorylus (Anomma) Stanleyi For.

$\sigma^{x}$ Congo (КонL).

Dorylus (Anomma) opacus For.

ot Congo (KонL).

Dorylus (Anomma) Emeryi Mayr.

$\Varangle$ Congo (KoHL), reçu par M. Wasmann. Ghez l'opacus $\Varangle$, la tète est plus plate que chez l'Emeryi.

Dorylus (Anomma) Kohli Wasm. v. Frenisyi n. var.

§. Longueur : $4^{\mathrm{mm}}-8^{\mathrm{mm}}, 5$. Plus petit que la forme typique et surtout beaucoup plus mat, surtout la tête qui est à peine un peu subopaque, presque mate. La tête est aussi légèrement plus plate avec les còtés un peu plus droits que chez le type. Congo (КонL).

Dorylus brenipennis Em. v. Marschalli Em.

¡ Congo (Kohl, reçu par M. Wasmans). 


\section{$3^{\text {me }}$ Sous-famille Myrmicinae Lep.}

Sima (Pachysima) aethiops Sm.

$\Varangle$ ㅇ. Watikaia sur le fleuve Chopo, Candolo sur l'OkiavoLindi, dans les branches creuses d'une plante myrmécophile (Barberia fistulosa Mast.).

Sima Oberbecki For.

q Leopoldville, Bas-Congo (KoнL), dans la même plante que S. (Pachysima) aethiops.

q non encore décrite. Longueur: $7^{\mathrm{mm}}$. Mésonotum à peine plus large que le reste. Les ailes manquent. Du reste absolument semblable à l'ouvrière, mais la tête est encore plus longue, $1^{2}{ }_{3}$ fois plus longue que large. Il s'agit d'une petite $q$ à peine aussi grande que l'ouvrière typique.

Sima Mocquerysi André.

ф Congo (Конц), même plante que les précédentes.

\section{Sima anthracina Sant.}

§ St-Gabriel, Congo (Kohl), même plante que les précédentes.

Sima Prelli For. v. odiosa n. var.

భ. Longueur: $6^{\mathrm{mm}}$. Diffère du type par sa couleur entièrement noire, avec les mandibules, les antennes et les pattes roussâtres, les cuisses brunes et le milieı des funicules obscurci. La tête est aussi un peu plus longue et les funicules légèrement plus grêles. Prise avec la Sima Mocquerysi, au même endroit.

\section{Sima ophthalmica Em.}

¡ Bengemeza et $\mathrm{S}^{\mathrm{t}}$-Gabriel, Congo (KoнL), même plante que les précédentes; élève des Pucerons.

Les $\Varangle$ sont un peu plus grandes que le type, d'environ $4^{\mathrm{mm}}$. L'épinotum est fort bossu; ses deux faces passant de l'une à l'autre. Le pétiole est bas, étroit; son nœud également convexe, très peu distinct de son court pétiole antérieur et bien 2 fois 
plus longs que large. Le postpétiole plus large, cupiliforme, est un peu plus long que sa largeur postérieure.

Myrmicaria eumenoides Gerst.

¡ q Romé près Stanleyville Congo (КонL). M. KонL a aussi récolté la v. congolensis.

Myrmicaria exigua And.

Congo (КонL).

Myrmicaria exigua And. v. rufiventris For.

¡ Makanga Congo (KонL). Ici encore M. КонL a trouvé cette forme dans de petits nids sur les feuilles, mais il dit qu'ils sont faits avec une toile tissée ou filée, entremêlée de matériaux végétaux, et non en simple carton.

Cremastogaster concava Em.

$\Varangle \subsetneq \sigma^{x}$. Pris plusieurs fois à $\mathrm{S}^{t}$-Gabriel, etc., sur des nids de Termites, dans les cases supérieures d'appendices coniques, au haut des dits nids. Envoyé aussi par le prof. Wasmann.

Cremastogaster pauciseta Em. r. grossulior n. st.

广. Longueur : $2^{\text {mm }}, 9-3^{\text {mı }}, 2$. Diffère avant tout de l'espèce typique par sa taille bien plus large et bien plus robuste. La tête n'est pas rétrécie, ni convexe derrière les yeux comme chez l'espèce typique, mais elle est large avec un bord postérieur à peu près droit. Les scapes sont un peu plus courts, dépassant le bord postérieur seulement de leur épaisseur. Le thorax a à peu près la mème forme, mais il est plus robuste; l'échancrure mésoépinotale est moins profonde, la face basale de l'épinotum bien plus longue, avec des épines bien moins longues, plus courtes qu'elle et moins verticales. Thorax et pédicule bien moins luisants. Epinotum et pronotum subopaques, assez fortement réticulés. Couleur d'un jaune plus roussâtre, plus foncé, avec seulement la moitié postérieure de l'abdomen brune.

St-Gabriel, Congo (Конц), dans les nectaires floraux d'un buisson. Mon Cr. dolens (1910) n'est guère qu'une race de pauciseta Em., auquel je l'avais déjà comparé du reste. 
Cremastogaster luctans For. v. rugosior. Sant. ґ Congo (KонL).

Cremastogaster Ruspolii For. v. atriscapis For.

Pris une fois à $\mathrm{S}^{t}$-Gabriel, dans une plante myrmécophile, et une autre fois dans un grand nid de carton (aussi a St-Gabriel) appliqué sur une plante de Cacao où il cultivait des Coccides en grand, arrêtant ainsi le développement des fruits. Parfois les fruits étaient eux-mêmes recouverts de carton.

Cremastogaster nigeriensis Sant. v. wilniger $\mathrm{n}$. var.

ఢ. Longueur: $2^{\mathrm{mm}}-2^{\mathrm{mm}}, 1$. Plus petite que le type et de couleur plus foncée, brun clair, avec les ${ }^{2} / 3$ postérieurs de l'abdomen et la tête d'un brun foncé, la massue des antennes et les tarses d'un roux jaunâtre. Le reste des antennes et des pattes d'un brun clair. Les épines sont plus longues et bien plus pointues, de moitié plus courtes que la face basale. La tête est aussi plus étroite, un peu plus longue, plus rétrécie et plus convexe derrière; les articles de la massue sont un peu plus longs et plus grêles et le scape plus long. L'Insecte est moins lisse, la tête un peu subopaque, distinctement réticulée, de même que le thorax. Du reste identique.

․ Longueur: $8^{\text {mm }}, 1$. Mème couleur que l' $\not{q}$. Quelques faibles stries longitudinales sur la tête. Mésonotum lisse. Epinotum inerme, avec une courte face basale ridée en travers ét presque horizontale et une haute face déclive subverticale. Les ailes manquent. Tête rectangulaire, plutòt plus large que longue. Le scape atteint à peine le bord postérieur.

St-Gabriel, Congo (KoHL), dans une plante myrmécophile. Cette forme diffère du Willwerthi Sant., sa voisine, par la sculpture plus faible du thorax, par son pronolum sans angles et bien plus convexe, seulement subbordé. Elle me paraît néanmoins constituer un passage du nigeriensis au Willwerthi. Elle ressemble aussi au Muralti For., mais en diffère par son pétiole élargi devant, par son thorax étranglé, etc. 
Cremastogaster censor For. r. Junodi n. st.

ఢ. Longueur : $3^{\mathrm{mm}}, 3$. Plus petit que le type dont il diffère en outre par une carène longitudinale au milieu du mésonotum qui est un peu moins concave derrière. La tête est en outre un peu moins large et surtout subopaque, presque mate, avéc des rides longitudinales très serrées et fines, tandis qu'elle est lisse chez le type du censor. L'abdomen est par contre fort lisse, plus que chez le type. La couleur est la même, à part la tête qui est brune.

Récolté par M. Junod au Transvaal à Shilowana (Musée de Genève). Dans ma description du type j'ai oublié le caractère principal, c'est-à-dire la forme du thorax qui est toute différente du senegalensis dont j'en avais fait une race. Chez le senegalensis le promésonotum est lortement convexe en tous sens; chez le censor la moitié antérieure du pronotum est ascendante, passe par une courbe brusque au reste du pronolum et au mésonotun qui sont presque plats, à peine convexes. Par suite, l'épinotum est à la même hauteur que le mésonotum, séparé de lui seulement par une suture profonde et enfoncée. C'est pourquoi je crois devoir séparer le censor, comme espèce distincte, du senegalensis.

Cremastogaster australis Mayr v. sycites $\mathrm{n}$. var.

§. Longueur: $4^{\mathrm{mm}}, 2$. Bien plus petit que le type, avec les deux nouds plus étroils, le pétiole peu élargi devant et la couleur plus foncée, d'un bı'un roussâtre, avec le vertex et l'occiput bruns et l'abdomen noir.

Townsville, N. Queensland, reçu de M. Donisthorpe.

Cremastogaster vulcania Sant. r. Godefreyi For. v. foraminicipoides $\mathrm{n}$. var.

Longueur : $3^{\mathrm{mm}}, 8-4^{\mathrm{mm}}, 6$. Noir, mandibules, antennes et pattes brunes; tête subopaque, presque mate, très densément et finement striée, avec d'assez gros points enfoncés, rappelant ceux du foraminiceps Sant.

Bulawayo, Rhodesia (Arnold) (No 108). Je l'avais considéré dans le temps (Annales Soc. Ent. Belg. 1913, page 124) comme simple variété du vulcania, sans lui donner de nom. 
J'ai eu le tort de rattacher la r. Godefreyi au foraminiceps Sant., dont le pronotum bordé et presque concave en dessus a une toute autre forme. Les épines sont aussi bien plus courtes chez le Godefreyi. Du reste toutes ces espèces sont très voisines et pleines de transitions.

Cremastogaster Menileki For. r. Satan n. st.

§. Longueur: $3^{\mathrm{mm}}, 6-4^{\mathrm{mm}}, 1$. Sculpture et épinotum d'alulai Em., mais le mésonotum et le pronotum sont bien plus concaves, comme chez le type du Menileki. La couleur est d'un brun roussâtre, avec la tête presque noire. Les épines sont pointues et assez courtes, le pétiole en trapèze renversé, assez long et à còtés droits. Postpétiole petit, sillonné. La tête est lisse.

Congo (KоHL).

Cremastogaster Menileki For. r. Satan For. v. satanula n. var.

§. Longueur: $2^{\mathrm{mm}}, 4-2^{\mathrm{mm}}, 6$. Diffère du précédent, outre sa petite taille, par son abdomen presque noir, par ses scapes un peu plus longs, dépassant distinctement le bord postérieur, et par ses épines plus longues aussi.

Trouvé dans la fente d'une écorce d'arbre couverte d'une toile avec matériaux végétaux. S'-Gabriel, Congo (КонL). Les deux formes précédentes diffèrent de pronotalis Sant. par leur postpétiole sillonné et leur pétiole moins anguleux. D'après Santschi, le $C$. alulai serait une race de Menileki, tandis qu'Emery veut rattacher mon scrutans comme race à l'alulai. Le dédale des transitions est presque infini.

\section{Cremastogaster kasaiensis For.}

$\subsetneq$ S'-Gabriel et autres lieux, Congo, récolté par M. KoнL sous 28 numéros différents. Les ailes de la $q$ sont d'une couleur brune assez foncée, fort accentuée. L'énorme différence entre l' $\Varangle$ et la $q$ est partout la même ou peut s'en faut. L'ouvrière varie entre $2^{\mathrm{mm}}, 1$ et $3^{\mathrm{mm}}, 2$. Toutes les Fourmis ont été récoltées dans les internodium de plantes myrmécophiles. L'analogie du Cr. kasaiensis avec diverses races du Buchneri est telle que je suis obligé, malgré tout, d'en faire une simple race 
de cetle espèce. La $q$ surtout ressemble à s'y méprendre à celle de la r. Winkleri For. J'ai donc pensé qu'il pouvait s'agir de simples fourmilières commençantes, d'autant plus que M. КонL a trouvé souvent une $q$ seule avec les $\Varangle$. Néanmoins, dans 8 numéros différents, il m'a envoyé entre 50 et 100 ouvrières de chacun. L'un d'eux avait plusieurs . . Il est donc inadmissible que toutes ces fourmilières fussent commençantes et je dois maintenir le kasaiensis, au moins comme race. La taille de la $q$ varie entre $7^{\mathrm{mm}}, 8$ et $9^{\mathrm{mm}}, 3$.

Cremastogaster impressa Mayr sens. strict.

‘ $\sigma^{x}$ Kilongalonga, Congo (KонL), dans une tige creuse de Buchnacrodendron speciosum Gürke et dans d'autres plantes myrmécophiles à St-Gabriel.

$\sigma^{x}$ encore inédit. Longueur: $3^{\mathrm{mm}}, 6$. Mandibules formant une seule dent aiguë. Epinotum inerme. Pétiole en trapèze renversé fort élargi devant. Mésonolum densément strié en long. Entièrement noir, avec les antennes, les tibias et les tarses roussâtres, les cuisses brunes et les ailes subhyalines. Chez la $q$, la première moitié des ailes est brunâtre (un peu comme chez certains Lasius d'Europe). Cette espèce est très voisine de la forme que j'ai appelée $\mathrm{Cr}$. excisa, r. Andrei, surtout de sa variété gordonensis For. Ces formes constituent un passage de l'excisa à l'impressa.

\section{Cremastogaster impressa Em. v. sapora n. var.}

广. Longueur: $3^{\mathrm{mm}}, 6-4^{\mathrm{mm}}$. Diffère du type par son promésonotum subopaque et réticulé, sans rides longitudinales. Les épines sont beaucoup plus courtes, à peu près comme chez l'excisa v. Kohliella For. La couleur est variée de roux et de brun foncé sur le thorax; la tète est brune derrière, roussâtre devant. Du reste comme le type.

ๆ. Longueur: $5^{\mathrm{mm}}, 4$. Beaucoup plus petite que le type, avec les ailes presque hyalines (légèrement brunàtres (hez le type). L'épinotum a deux faibles dents obtuses. La tête est plus large que le thorax. Du reste comme le type. 
Congo (KонL). Cette forme fait un peu transition à l'excisa Mayr.

Cremastogaster excisa Mayr. r. Andrei For. v. Kohliella n. var.

§. Longueur : $2^{\mathrm{mm}}, 7-3^{\mathrm{mm}}, 6$. Très semblable au type de la race, mais les épines sont plus courtes, longues à peine comme $1 / 3$ de leur intervalle. La portion tronquée du mésonotum, derrière, est très abrupte, formant presque un angle droit avec le dos.

S'-Gabriel, Congo (KонL) dans des plantes myrmécophiles.

Cremastogaster Buchneri For. v. Graeteri n. var.

६. Longueur: $3^{m m}, 5-3^{m m}, 7$. Beaucoup plus petite que le type, auquel il est du reste fort semblable. La tête est un peu plus longue, distinctement plus longue que large et moins échancrée derrière. Epines plus courtes, longues comme à peine 1/3 de leur intervalle. La sculpture, surtout sur la tête, est plus superficielle, seulement subopaque. Du reste identique.

St-Gabriel, Congo (KонL), nid fait en carton.

Cremastogaster Buchneri For. r. Winkleri For.

$\Varangle$ Congo (Конц), dans une plante myrmécophile.

Cremastogaster Buchneri For. r. Winkleri For. v. Fickendeyi For.

¡ Leopoldville, Congo inférieur; St-Gabriel, etc., Congo (KонL) avec des Coccides, 1 fois vers des Termites, nichant autour des plantes.

En outre M. Koнl a récolté diverses formes intermédiaires entre la r. Winkleri et la v. Fickendeyi, une fois dans un nid, probablement volé, de Tetramorium aculeatum, d'autres fois dans des plantes myrmécophiles, ou dans des nids de Termiles. Le $\mathrm{n}^{\circ} 68$ porte la très intéressante remarque suivante: « Fourmi " des plantes. Vit dans et sur la plante myrmécophile Plectronia " laurentii de Wilde. A 5 mètres du sol, se trouvait un tronc " portant le nid en carton de la Fourmi qui avait 40 à $50 \mathrm{~cm}$. de 
" haut. Mais la Fourmi habite en même temps toutes les bran" ches creuses de la plante. Makanga sur le fleuve Okiavo." De là, on peut conclure qu'il n'y a pas contraste absolu entre les nids en carton du Buchneri et l'habitat des tiges creuses.

Cremastogaster Buchneri For. r. Winkleri For. v. transversiruginota $\mathrm{n}$. var.

భ. Longueur: $3^{\mathrm{mm}}, 2-4^{\mathrm{mm}}$. Aussi grand que les plus grands Winkleri, dont elle se distingue par son mésonotum bien plus fortement tronqué derrière (presque comme chez l'excisa $\mathrm{r}$. Andrei), et dont la carène s'arrête derrière, vers le milieu. Puis, la face basale de l'épinotum a des rides transversales grossières. Le pétiole est un peu plus large devant; tout le thorax est un peu plus robuste.

Motombe sur le fleuve Okiavo. Pris dans un nid de carton de $50 \mathrm{~cm}$. de haut. Cette forme ressemble beaucoup à I'impressiceps Mayr v. Lujana For., mais ce dernier a le promésonotum fort et uniformément convexe, plus convexe mème que l'impressiceps typique, et sans carène.

Cremastogaster Buchneri For. r. Laurenti For. v. theta For.

$\Varangle$ St-Gabriel, Congo (Конц), dans les buissons. Une fois déménageant la nuit de nids de carton qui se trouvaient sur un arbre coupé.

ㅇ (encore inédite). Longueur: $5^{\mathrm{mm}}, 7-6^{\mathrm{mm}}$. Brune, avec les pattes, les antennes et les mandibules roussâtres. Scutellum et postsculellum verticalement tronqués, face déclive de l'épinotum de même. La face basale, courte et oblique, se termine par 2 épines pointues plus longues que la largeur de leur base. Pétiole presque circulaire. Postpétiole sans sillon distinct (distinct che\% l' $\dot{q})$. Luisant et presque lisse, sauf quelques rides superficielles sur la tête. Celle-ci bien plus large que longue.

Cremastogaster Buchneri For. r. africana Mayr v. Camena n. var.

ॐ. Longueur: $3^{\mathrm{mm}}, 3-3^{\mathrm{mm}}, 7$. Très près de la var. variegata 
Mayr, dont elle se distingue par sa couleur un peu plus foncée, sa taille un peu plus grande, puis surtout par ses épines bien plus courtes, subdentiformes, très épaisses à la base, par la carène plus nette du mésonotum et par la suture promésonotale plus enfoncée, faisant un peu feston de còté. Même sculpture que variegata. Chez le type de la r. Laurenti For. la suture promésonotale est aussi enfoncée que chez la var. biemarginata For. de la r. africana.

Olombo, Congo (КонL).

Si je récapitule l'étude précédente, j'en tire la conclusion qu'il y a là un dédale de formes transitoires qui, à mon avis, nous forcera de réunir les espèces suivantes comme races au $\mathrm{Cr}$. Buchneri For. : kasaiensis For., vulcania Santschi, impressiceps Mayr, excisa Mayr, impressa Em., Kohli For., Alulai Em. et même Menileki For. Peut-ètre même devra-t-on aller plus loin, mais pour le moment je m'arrête. En effet, la longueur des épines, la forme et la carène du mésonotum, la forme du pronotum, celle du pétiole et du postpétiole varient tellement que les caractères qu'on a donnés autrefois comme spécifiques ne tiennent plus debout. Les mœurs cartonnières et internodiales, nous venons de le prouver, montrent aussi des transitions.

Cremastogaster (Atopogyne) Luciae For.

o Congo (KонL).

Cremastogaster (Oxygyne) Stadelmanni Mayr.

$\Varangle$ St-Gabriel, Congo (KoHL), dans de grands nids de cartons contre les troncs d'arbres.

Cremastogaster (Decacrema) solenopsisoides Em. r. flavida Mayr v. convexiclypea $\mathrm{n}$. var.

ఢ. Longueur: $2^{\mathrm{mm}}-2^{\mathrm{mm}}, 5$. Se distingue du type de la race par son épistome beaucoup plus convexe et par sa couleur encore beaucoup plus pâle, d'un jaunâtre terne. Le mésonotum est aussi fortement creusé en long d'un bout à l'autre entre les 2 arètes, plus que chez le type. Les épines sont un peu plus longues. Du reste identique. 
O Longueur: $4^{\mathrm{mm}}, 6$. Antennes de 10 articles, comme chez l' $̛$. Ailes faiblement teintées de jaunâtre, à nervures pâles. Tète bien plus longue que large; le scape n'alteint que son cinquième postérieur. Massue moins distinctement biarticulée que chez l'ouvrière. Thorax plus étroit que la tête. Deux épines assez pointues à l'épinotum. Couleur d'un jaune un peu plus brunâtre que chez l'ouvrière.

$\sigma^{7}$ Longueur : $1^{\mathrm{mm}}, 5-\mathrm{L}^{\mathrm{mm}}, 9$. Mandibules très courtes, ne se rejoignant pas, obtuses à l'extrémité. Thorax un peu plus large que la tête. D'un jaune brunâtre sale; pattes et antennes jaune clair et pâle. Du reste comme la ㅇ. .

St-Gabriel, Congo (Конц). Dans une plante myrmécophile.

Cremastogaster (Decacrema) petiolidens n. sp.

§. Longueur : $1^{\mathrm{mm}}, 6-2^{\mathrm{mm}}$. Mandibules étroites, lisses, luisantes, ponctuées. Tête rectangulaire distinctement plus longue que large (d' $1 / 5$ environ), avec les còtés convexes et le bord postérieur un peu convexe aussi et plus étroit que le bord antérieur. Epistome court, un peu déprimé dans le sens longitudinal. Les yeux, petits et plats, situés à peine en avant des côtés, n'ont qu'une dizaine de facettes. Scape court, distant du bord postérieur d'environ 2 fois son épaisseur. Massue épaisse, de 2 articles. Funicule de 9 articles; dont les $2^{\text {me }}$ et $3^{\text {me }}$ peu distinctement séparés; articles 3 à 5 deux fois plus épais que longs. Pronotum assez distinctement épaulé. Sutures distinctes. Le pronotum a, entre son cou et son dos, un escalier antérieur fort abrupt. Mésonotum faiblement, mais distinctement convexe, nullement excavé, ni en long, ni derrière. Pas d'èchancrure mésoépinotale marquée, mais simplement un assez fort enfoncement de la suture. Face basale de l'épinotum un peu plus large que longue, convexe devant, concave entre les deux épines. Celles-ci sont triangulaires, à base très large, à peine ou pas plus longue que cette dernière, constituant deux très larges dents pointues. Face déclive plus courte que la basale. Pétiole carré, à peine ou pas plus large devant que derrière, muni de 4 dents; les antérieures petites, mais les postérieures très appa- 
rentes, pointues et fortes. Le postpétiole très étroit, avec un sillon médian étroit, mais fort distinct. Pattes courtes.

Lisse et luisant, avec une fine ponctuation éparse. Quelques fines stries sur le devant de la tête et quelques réticulations sur l'épinotum. Pilosité dressée extrêmement éparse et très courte. Pubescence très courte et diluée. Roussâtre ou rougeâtre avec la tête et l'abdomen bruns.

Congo (KонL), sans remarques spéciales. Cette curieuse espèce diffère du solenopsisoides par la forme du thorax et du pétiole, par ses petits yeux, etc. Elle ressemble aussi au Muralti For., mais celui-ci a 11 articles, pas de dents au pétiole, le postpétiole sans sillon, la tête toule autre, etc.

Pheidole speculifera Em. v. cubangensis For.

24. Vers le poste de Lisala au dessus de Nouvel-Anvers sur le Congo. Cette variété est plus près du type de l'espèce que la race crassinoda Em., à laquelle je l'avais autrefois rapportée.

\section{Pheidole Bucholzi Mayr.}

$\Varangle$ ○ Makanga, Congo (KoHL), chez des Termites, reçue aussi par M. Wasmann avec la $q$.

ㅇ (encore inédite). Longueur : $8^{\mathrm{mm}}, 6$. Mandibules luisantes grossièrement striées et poncluées, à bord externe presque droit. Tête un peu plus large que le thorax, concave derrière et grossièrement ridée en long, les rides divergeant en arrière. Le scape atteint le $5^{\text {me }}$ postérieur de la tête. Mésonotum et scutellum un peu moins grossièrement ridés en long que la tête, finement réticulés et subopaques comme elle. Epinotum ridé en travers avec de larges épines. Postpétiole un peu plus large que long, 3 fois plus large que le pétiole, rhombiforme, ridé en travers et subopaque. Premier segment de l'abdomen subopaque, densément réticulé. Les ailes manquent. Couleur d'un roussâtre uniforme. Du reste comme le 2 .

Pheidole caffra Em. r. Bayeri n. st.

2 . Longueur: $5^{\mathrm{mm}}$. Tête plus petite que chez la var. amoena

Rev. Suisse de Zool. T. 24. 1916. 
For., un peu plus longue, plus étroite derrière, où elle est plutòt moins large qu'au milieu. Elle est aussi moins déprimée à l'occiput, à peu près comme chez la r. abyssinica For. Le scape est plus long que chez toutes deux; il atteint presque le tiers postérieur de la tête. Le thorax est aussi plus grêle que chez les deux autres formes citées; le tubercule supérieur du pronotum est plus faible. Par contre le mésonotum est plus profondément enfoncé au milieu, présentant un bourrelei antérieur et un postérieur fort élevés. Face basale de l'épinotum bien plus longue que large, plus étroite que chez amoena et abysinica; épines subverticales, assez grêles, pointues, longues comme les $2 / 3$ de leur intervalle. Postpétiole un peu plus long que chez la r. abyssinica, mais à peine plus étroit; du reste conformé de même. Le pétiole est un peu plus long et plus étroit. La tête est luisante avec des rides très régulières sur le front où elles ne sont pas réticulaires; elles le deviennent sur les còtés et surtout sur l'occiput où elles divergent et qui n'est nulle part lisse. Ces rides sont plus grossières, mais bien moins serrées que chez la r. abyssinica. En outre, l'occiput a derrière une large impression médiane qui est à peine sensible chez l'abyssinica. Thorax ridé en travers, abdomen lisse. Pilosité dressée roussâtre et éparse. Scapes et tibias avec une longue pubescence adjacente. D'un brun foncé presque noir. Mandibules, scapes et cuisses bruns. Funicules, tarses et articulations roussâtres.

ఢ. Longueur: $2^{\mathrm{mm}}, 7$. Tête bien plus longue que large, plus longue que chez abyssinica. Elle est surtout plus rétrécie et convexe derrière, formant à peine un bord postérieur distinct. Le scape dépasse la tête des ${ }^{2} / 5$ de sa longueur (d'un tiers chez abyssinica). Mésonotum et épinotum comme chez le 4 , plus allongés que chez abyssinica, de même que les deux nœuds du pédicule. Lisse avec quelques stries obliques sur les joues; épinotum réticulé. Presque noire, mandibules jaunâtres, membrès comme chez le 24 , ainsi que la pilosité.

Kasindi, Congo ( ${ }^{r}$ Bayer), reçu du Musée de Bruxelles par M. Schouteden. 
Pheidole Aurivillii Mayr r. kasaiensis For.

భ, 2, $q$ Congo (Конц).

$\uparrow$ (encore inédite). Longueur: $5^{\mathrm{mm}}, 1$. Relativement petite. Mandibules luisantes, ponctuées, striées à la base. Tête en trapèze, rétrécie devant, un peu plus large derrière que longue, à bord postérieur droit. Thorax court à dos aplati. Abdomen tronqué devant. Tête plutòt luisante, grossièrement ridée en long. Sauf le devant du scutellum, tout le reste est mat, le thorax densément ridé et l'abdomen densément réticulé-ponctué. De gros points piligères, surtout sur l'abdomen. Pilosité et pubescence comme chez le 2 . D'un noir brunâtre; cuisses brunes au milieu; le reste des membres, les mandibules et une tache à l'angle antérieur de l'abdomen, roussâtres. La ㅇ a été trouvée à St-Gabriel, dans un nid de Termites abandonné.

\section{Pheidole megacephala F. r. nkomoana n. st.}

24. Longueur: $2^{\mathrm{mm}}, 6-3^{\mathrm{mm}}, 1$. Mandibules avec une fine ponctuation plus abondante que chez le type de l'espèce. La tête est relativement plus petite que chez le type; ses còtés sont moins convexes et ses angles occipitaux bien plus aigus, laissant entre eux deux une concavité plus grande. Les yeux sont plus convexes et le scape est plus long, atteignant le tiers postérieur. Le promésonotum est moins convexe, avec des tubercules supérieurs plus accentués et une impression transversale nette au milieu du mésonotum. Les pattes et les antennes (massue y comprise) sont sensiblement plus grèles. Les arêtes frontales se prolongent jusqu'au quart postérieur de la tête, laissant en dehors un espace un peu plus lisse, et les rides longitudinales vont même un peu plus loin, jusqu'au $5^{\text {me }}$ postérieur. Pilosité un peu plus longue, plus espacée et plus fine. Epinotum plus luisant et plus lisse. D'un roux jaunâtre. Pattes et antennes jaunes. Pédicule et têle d'un roux un peu brunâtre. Milieu de l'abdomen brun. La couleur varie du reste; souvent la tète est brune et l'abdomen jaune-brunâtre. Tout le reste est comme chez le type de l'espèce.

ఢ. Longueur: $1^{\mathrm{mm}}, 8-2^{\mathrm{mm}}, 2$. Tête rétrécie et convexe derrière 
avec un bord postérieur indistinct, fort étroit, un peu moins convexe. Yeux fort convexes. Le scape dépasse l'occiput de plus d'un tiers de sa longueur. Promésonotum moins convexe que chez le type, avec une impression transversale indistincte au mésonotum. Face basale de l'épinotum 2 fois plus longue que large. Nœud du pédicule, comme tout l'Insecte, plus allongé que chez le type. Membres, pilosité et pubescence comme chez le 2 , de même que les variations de couleur. Sculpture comme chez le type, un peu plus lisse.

q. Longueur $5^{\mathrm{mm}}$. Tète moins rétrécie devant et plus concave derrière que chez le type. Mandibules comme chez le 2 . Arêtes frontales très distinctement prolongées jusqu'au $5^{\text {me }}$ postérieur de la tête, laissant en dehors un espace lisse. Postpétiole 2 fois plus large que long. Rides de la tête comme chez le type, mais un peu plus lâches. Pilosité, pubescence et couleur comme chez le 4 .

$\sigma^{x}$. Longueur: $3^{\mathrm{mm}}, 5$. Les yeux fort grands atteignent presque le bord antérieur de la tête en surplombant un peu ses còtés. Thorax beaucoup moins large que chez le type. Ailes faiblement jaunâtres, avec des nervures assez pâles. Les ocelles sont beaucoup moins grands que chez le type et la tête est plus prolongée derrière entre les yeux. Brunâtre, mandibules, antennes et pattes jaunâtres.

St-Gabriel, Congo (КонL). Trouvé plusieurs fois sous 6 numéros différents, toujours dans des plantes myrmécophiles, tandis que M. KonL a trouvé plusieurs fois les Pheidole megacephala et punctulata, mais avec leur autre habitat bien connu. Cette race diffère des r. Santschii et atrox par la forme de l'occiput. Elle se rapproche un peu de la r. Gietleni For. de Madagascar, mais en diffère aussi. J'ai reçu par M. Wasmans la même race, mais d'une variété un peu moins différente de l'espèce typique. La r. nkomoana mérite peut-être de constituer une espèce distincte.

Pheidole rotundata For. r. impressifrons Wasm.

४, ‘ Congo (KoHL), reçue par M. Wasmann. 
Je maintiens mon sous-genre Allopheidole contre l'opinion de M. EMERy.

Oligomyrmex angolensis Sant. r. congolensis n. st.

2 . Longueur : $1^{\mathrm{mm}}, 4-1^{\mathrm{mm}}, 5$. Un peu plus grand que le type ; sculpture identique, ainsi que.la forme de la tête, mais celle-ci a une très petite crête, de chaque còté, sur l'occiput. Le thorax n'a par contre presque pas d'échancrure. L'épistome est fortement échancré au milieu de son bord antérieur, mais il n’a pas les carènes latérales que dessine et décrit Santschi chez le type. La tête est subopaque et densément striée devant. Le reste du corps est assez luisant et peu sculpté, d'un jaune terne et sale. Tète d'un jaune brunâtre, antennes et pattes jaunes. L'extrémité du scape dépasse à peine le milieu de la tête. Les dents de l'épinotum sont plus courtes que chez le type, petites et triangulaires.

ఢ. Longueur: $0^{\mathrm{m} n}, 9$. Sculpture de la tète, qui est mate, un peu plus réticulaire et moins striée que chez le 2 . Le scape atteint le quart postérieur. Echancrure mésoépinotale un peu plus forte, les dents de l'épinotum aussi. Le pétiole est plus épais et plus arrondi au sommet que chez le 2 où son nœud paraît plus haut et plus comprimé que chez le type. D'un jaune un peu roussâtre assez uniforme, avec les pattes jaunes.

Congo (KонL). Cette race diffère de Jeanneli Sant., qui a la tête lisse, par ses còtés convexes et non droits. La tête du 2 est bien plus longue que chez Alluaudi, mais moins longue que chez Jeanneli; elle est un peu intermédiaire entre angolensis s. str. et Alluaudi.

Monomorium albopilosum Em. v. Thales For.

§ Shilowana, Transvaal, récolté par M. Junod et reçu du Musée de Genève.

La v. diserta For. du M. Salomonis doit ètre rapportée à la r. termitarium et non pas au Salomonis s. str. Le Monomorium Schultzei For. est très rapproché de la race termitarium For. du Salamonis L. 
Monomorium Oscaris For. r. springvalense, v. paterna For.

§ St-Gabriel, Congo (КонL), dans une plante myrmécophile. Cette forme ressemble aussi assez au M. Schultzei For.

Monomorium rhopalocerum Em. v. gabrielensis n. var.

ఢ. Longueur: $1^{\mathrm{mm}}, 2-1^{m+m}, 4$. Diffère du type par le nœud du pétiole plus court, bien plus comprimé d'avant en arrière, plus pointu au sommet et aussi moins large. L'échancure du thorax est encore plus profonde. Du reste comme le type.

ㅇ. Longueur: $3^{m m}, 2$. La tête a les côtés convexes ainsi que le bord postérieur; elle est presque aussi large derrière que longue. Le scape dépasse le bord postérieur d'à peine son épaisseur. Thorax court et fort convexe. Pilosité du corps fine et assez abondante. Celle des membres, subadjacente. Pétiole comme chez l'ouvrière. Jaunâtre, avec l'abdomen d'un brun foncé, tronqué devant. Les ailes manquent.

St-Gabriel, Congo (КонL), dans un petit nid de Termite.

Monomorium (Mitara) exiguum For. r. flavescens $\mathrm{n}$. st.

广. Longueur: $1^{\mathrm{mm}}-1^{\mathrm{mm}}, 3$. Entièrement d'un jaune sale aver la tête un peu plus foncée, d'un jaune un peu brunâtre. La tète est plus allongée et plus rectangulaire que chez le type, d'un tiers plus longue que large. Les scapes, plus courts, n'atteignent que le tiers postérieur de la tête. L'étranglement du thorax est sensiblement plus fort que chez le type qui est presque noir, comme la var. bulawayensis For.

St-Gabriel, Congo (КонL), pris trois fois dans des plantes myrmécophiles.

J'ai décrit la var. bulawayensis en 1913. Comme j'ai décrit sous le même nom un Monomorium amblyops Em. r. bulawayense en 1914, et que dernièrement on commence (je pense avec raison) à interdire l'emploi du même nom pour les variétés du même genre, je me permets de changer le nom précédent en $M$. amblyops Em. r. Prossae n. st.

Podomyrma Delbrücki For.

克 Townsville, Nord Queensland, envoyé par M. Donisthorpe. 
C'est une variété chez laquelle les dents du pronotum sont un peu plus fortes et pointues et où les còtés du mésonotum ont aussi une protubérance dentiforme plus nette. Je ne lui donne pas de nom spécial.

Atopomyrmex Mocquerysi André var. curvispina For.

Lumbulumbu, Congo (КонL), courant sur un tronc d'arbre.

Rhoptromyrmex Rothneyi For. v. intermedia For.

¡ MontMatang, Sarawak (G.-E. Bryant), envoyé par M. DonisTHORPE.

Rhoptromyrmex opacus Em.

¡ Congo (KoHL).

Rhoptromyrmex globulinodis Mayr r. Alberti n. st.

ఢ. Longueur: $2^{\mathrm{mm}}, 1-2^{\mathrm{mm}}, 5$. Diffère du type de Mayr par son occiput bien moins concave, par les côtés de la tête moins convexes, le scape un peu plus long, le pétiole plus étroit et la couleur d'un jaune roussâtre avec les membres jaunes. Mais il est lisse et luisant comme le type, ce qui le distingue d'opacus.

Congo (KоHL).

Tetramorium guineense F. r. Peutli n. st.

భ. Longueur: $2^{\mathrm{mm}}, 4-2^{\mathrm{mm}}, 6$. Ressemble à la v. Phasias For., mais elle s'en distingue, et bien plus encore du type de l'espèce, par la forme du nœud du pétiole. Celui-ci est plus bas et plus arrondi devant, comprimé en toit au sommet, presque sous forme d'arète s'élevant en arrière. Sa face postérieure est un peu concave, du reste abruptement tronquée. Les épines sont larges, fortes, recourbées en avant. La sculpture est plus grossière que chez la var. Phasias, plus semblable à celle du type de l'espèce, mais la tête est plus réticulée et moins ridée. Couleur comme chez le type de l'espèce, mais la Fourmi est bien plus petite. Les articles 2 à 5 du funicule sont très courts et très petits.

․ Longueur: $3^{\mathrm{mm}}, 1$. Beaucoup plus petite que le type de guineense. Comme du reste chez l'ouvrière, les arêtes frontales, prolongées jusqu'au $10^{\text {me }}$ postérieur de la tête, laissent entre 
elles et les yeux presque un demi-scrobe, ou, si l'on veut, un scrobe superficiel avec faible sculpture. La tète est rectangulaire, d'un quart plus longue que large. Les mandibules sont, comme chez l'ouvrière, lisses et luisantes avec des points épars et très fins (subopaques et sculptées chez le type, luisantes, mais bien plus ponctuées chez la v. Phasias). La tête est aussi large à son quart antérieur que derrière, un peu plus large que le thorax. Epines très épaisses, peu courbées en avant. Les épines inférieures de l'épinotum ne sont pas beaucoup plus courtes; elles sont translucides et courbées en avant. Ailes un peu jaunàtres, à nervures pâles. Tout le reste, en particulier le pétiole, comme chez l'ouvrière. La pilosité et la pubescence de l'ouvrière et de la $q$ sont comme chez le type.

Congo (Kohl), reçu par M. Wasmann.

Tetramorium aculeatum Mayr.

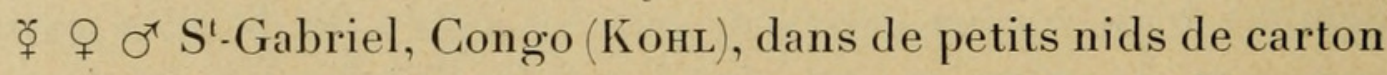
construits sous une feuille, souvent très fréquents sur le même arbre, jusquà 50 ou plus. Cette espèce aime les bas-fonds humides. Trouvé aussi par M. КонL au Poste de Lisala et ailleurs.

Tetramorium aculeatum Mayr v. major For.

ґ. † St-Gabriel, Congo (КонL), nids plus grands que chez le type.

Tetramorium aculeatum Mayr v. Wasmanni For.

‘ $\mathrm{S}^{\mathrm{t}}$-Gabriel, Congo (KонL), nichant comme le type sur une feuille de Palmier.

Tetramorium aculeatum Mayr v. rubroflava n. var.

భ. Longueur: $2^{\mathrm{mm}}, 6-3^{\mathrm{mm}} 1$. Tout semblable au type de l'espèce, dont il a aussi les longues épines et toute la forme du corps. Néanmoins, les épines sont moins divergentes, le bord postérieur de la tête est un peu plus distinct et les membres sont légèrement plus grèles, le scape dépassant le bord postérieur d'un peu plus du tiers de sa longueur. La sculpture est aussi un peu plus fine et un peu plus serrée. Couleur entière- 
ment d'un jaune à peine roussàtre. Quelquefois la tête, le prothorax et les cuisses un peu brunâtres.

$\mathrm{S}^{t}$-Gabriel, Congo (Конц), trouvé dans des nids tissés en soie, fixés sur les feuilles, et que M. KонL dit ètre semblables à ceux des Oecophylla et des Polyriachis. De ce fait, je conclus que le carton des nids du T. aculeatum n'est probablement que superposé à une toile filée (combinaison de carton et de toile), comme je l'ai prouvé pour beaucoup de Polyrhachis.

Tetramorium africanum Mayr.

¡ St-Gabriel, Congo (Конц). Construit des nids semblables à ceux du T. aculeatum, mais beaucoup plus grands; sa piquire est horrible, pire que celle de la Pachysima aethiops, et on la craint beaucoup.

Tetramorium sericeiventre Em. r. inversa Sant.

६ S'-Gabriel, Congo (KонL).

Tetramorium camerunense Mayr v. Gegaimi n. v.

భ. Longueur : $1^{\mathrm{mm}}, 8-2^{\mathrm{mm}}$. D'un roux jaunàtre clair, avec les membres jaunes, le postpétiole et l'abdomen bruns. Les stries de la tête sont aussi plus serrées que chez le type de l'espèce.

ㅇ. Longueur: $2^{\mathrm{mm}}, 6$. Tête un peu plus large que le thorax. Scape distant du bord postérieur de son épaisseur. Mésonotum et scutellum régulièrement striés en long. Le mésonotum est bordé sur le dos de chaque còté par une arête noirâtre qui n'existe pas chez l'ouvrière. Face basale de l'épinotum ridée en travers. Epines fortes, aussi longues que leur intervalle. Couleur comme chez l'ouvrière, mais la tête et le thorax plus foncés, roussâtres; le thorax varié de brun. Les ailes manquent.

St-Gabriel, Congo (КонL), dans un nid de Termites abandonné.

\section{Tetramorium simillimum $\mathrm{Sm}$.}

¡ $\sigma^{x} \mathrm{~S}^{t}-$ Gabriel, Congo (KoHL), sous une tuile. En comparant le type de la $q$ du Tetramorium pygmaeum Em., que m'a donné dans le temps M. Emery, je la trouve absolument identique à la $\odot$ du simillimum, et par conséquent synonyme. 
Tetramorium simillimum Sm. $r$. isipingense For. v. Dumezi n. var.

భ. Longueur: $2^{\mathrm{mm}}, 1-2^{\mathrm{mm}}, 5$. Plus petit que le type de la race. Les dents de l'épinotum sont un peu plus fortes et plus distinctes. Le nœud du pétiole est un peu moins arrondi. Le scape de l'antenne est plus court, distant du bord postérieur, qui est plus concave, de $2 \frac{1 / 2}{2}$ fois son épaisseur au moins. Le demiscrobe en dehors des arêtes frontales est aussi notablement plus net. Toute la Fourmi a une stature un peu plus robuste. Couleur un peu plus foncée sur la tête, mais plus claire sur l'abdomen.

St-Gabriel, Congo (КонL) dans une plante myrmécophile.

\section{Tetramorium Meressei $\mathrm{n}$. sp.}

广. Longueur: $2^{\mathrm{mm}}, 6-2^{\mathrm{mm}}, 9$. Aussi grand que la r. isipingense ci-dessus. Mandibules luisantes, faiblement ponctuées, à 6 ou 7 dents indistinctes; épistome caréné au milieu, à bord antérieur à peine ou pas échancré. Tête absolument rectangulaire, d'un quart à peine plus longue que large, à bord postérieur faiblement échancré au milieu. Yeux convexes, au milieu des côtés. Arêtes fronlales atteignant presque le bord postérieur de la tête, laissant en dehors un demi-scrobe à sculpture plus faible. Une arête latérale distincte est entre les yeux et le bord antérieur de la tête. Le scape atteint environ le $10^{\text {me }}$ postérieur de la tête. Suture promésonotale obsolète. Angles antérieurs du pronotum obtus, mais assez marqués. Promésonotum médiocrement convexe en tout sens. Suture mésoépinotale pas très distincte; étranglement mésoépinotal très faible, tout juste visible. Face basale de l'épinotum, un peu moins de 2 fois plus longue que large, sans bord distinct, bien plus longue que la face déclive, qui est bordée et terminée par 2 petites dents triangulaires. Celles-ci sont un peu plus courtes que les dents inférieurs de l'épinotum qui sont aussi plus pointues. Pétiole muni en dessous d'une petite dent oblique; son nœud, à peine plus long que le pédicule antérieur, est un peu plus haut et surtout encore beaucoup plus arrondi, devant, derrière et dessus que chez le simillimum r. isipingense 
chez lequel il est encore assez nettement cubique. Postpétiole court, encore plus haut que le nœud du pétiole, un peu plus large que lui, aussi large que long, 1 1/2 fois plus haut que long, verticalement tronqué derrière.

Luisant. Tête et thorax assez grossièrement ridés en long, les rides régulières sur le front, plus réticulées ailleurs. Nœuds du pétiole et du postpétiole assez grossièrement et obliquement réticulés de còté, lisses derrière; abdomen lisse, avec une ponctuation piligère abondante et très distincte. Le nœud du pétiole est comprimé en haut en forme de toit, comme chez le guineense r. Peutli, quoiqu'il ait du reste une autre forme; le sommet du postpétiole est aussi rétréci. Tout le corps et les membres sont recouverts d'une abondante pilosité très fine, jaune pâle, longue, d'aspect laineux, plus courte sur les membres, mais nullement polyfide. D'un roux un peu brunâtre. Pattes, antennes et mandibules jaunàtres; milieu de l'abdomen indistinctement brun.

Congo (KонL). Cette espèce a une fausse ressemblance avec le guineense r. Peulti. Sa carène latérale des joues, sa pilosité et ses deux nœuds sont très particuliers.

Triglyphothrix gabonensis André v. Boulognei n. var.

. Longueur : $3^{\mathrm{mm}}, 5-3^{\mathrm{mm}}, 8$. Se distingue du type de l'espèce par les nœuds du pétiole et du postpétiole qui sont tous deux moins larges; en outre, la sculpture de la tête, du thorax et des nœuds est plus forte et bien plus rugueuse, les rendant subopaques (le type est assez luisant et plutòt ponctué). La couleur est aussi plus foncée, d'un brun foncé, avec les membres et les mandibules roussâtres.

Congo (KонL).

Leptothorax (Goniothorax) Evelynae n. sp.

ఢ. Longueur : $2^{\mathrm{mm}}, 3-2^{\mathrm{mm}}, 5$. Mandibules assez luisantes, très finement ridées ou réticulées, armées de 4 dents. Epistome sans carène, avancé fortement devant en lobe arrondi, avec le bord entier. Tète rectangulaire, de presque $1 / 4$ plus longue que large, distinctement rétrécie d'arrière en avant, à bord postérieur et à 
còtés presque droits, avec l'angle postérieur arrondi. Yeux plutòt grands, au milieu. Le scape atteint presque le bord postérieur. Dernier article du funicule 3 fois plus long que l'avant-dernier; ses articles 2 à 8 presque deux fois plus épais que longs. Angles antérieurs du pronotum distinctement anguleux, mais pas dentiformes. Thorax subbordé latéralement. Suture promésonotale obsolète. Promésonotum faiblement convexe. Le mésonotum a une dent latérale obtuse à son quart postérieur. Echancrure mésoépinotale très faible en tout sens, presque nulle sur le dos, mais la suture est distincle. Face basale $1 \frac{2}{3}$ fois plus longue que large. Epines un peu obtuses, subhorizontales, peu divergentes, aussi longues que leur intervalle. Face déclive plus courte que la basale, assez bordée, munie en bas d'une dent triangulaire. Nœud du pétiole presque conique, mais arrondi au sommet. Sa base est 2 fois plus longue que le pédicule antérieur et son sommet étroit est néanmoins $1^{1 / 2}$ fois plus large que long. Postpétiole presque 2 fois plus large que long et que le nœud du pétiole, un peu plus bas que ce dernier. néanmoins un peu plus haut que long. Les deux nœuds sont arrondis en tout sens. Cuisses un peu renflées au milieu.

Subopaque. Tête parsemée d'assez gros points, fossettes très abondants, mais fort superficiels et en outre, dans les points comme ailleurs, extrèmement finement et faiblement réticulés. Ça et là, surtout derant, quelques stries longitudinales. Le thorax et les deux nouds ont la même sculpture fine, mais, au lieu de gros points, le promésonotum a d'assez grosses rides longitudinales superficielles et la face déclive de l'épinotum des rides transversales. Abdomen lisse et luisant avec des points épars, finement réticulé et un peu subopaque devant; de même les pattes et les scapes. Pilosité sétiforme obtuse un peu plus longue et moins abondante que chez l'innocens For., nulle sur les membres. Pubescence jaunâtre claire fort distincte et adjacente sur le corps et sur les membres. Entièrement d'un jaune très terne, à peine teinté de brunâtre, avec l'abdomen et les membres plus clairs, fort pâles.

우. Longueur: $2^{\mathrm{mm}}, 7$. Absolument semblable à l'ouvrière. 
Tête à peine plus large que le thorax. Mésonotum distinctement ridé en long; rides assez serrées. Ailes un peu jaunâtres, à tache marginale brune. Tout le reste, aussi la couleur, comme chez l'ouvrière.

S'-Gabriel, Congo (KонL) dans une plante myrmécophile.

Cette espèce, très semblable à ce que j'avais appelé Tetramorium (Leptothorax?) innocens, me prouve définitivement que cette dernière forme était un Leptothorax et non pas un Tetramorium. L'Evelynae diffère de l'innocens par la forme tout autre du noud de son pétiole bien plus mince (plus court) et plus arrondi au sommet. Les épines et les scapes sont plus longs, l'échancrure thoracique plus faible, la couleur analogue. Si M. Kонц n'avait pas prouvé le contraire, on serait tenté d'attribuer à l'innocens et à l'Evelynae des mœurs psammophiles analogues à celles du groupe Laurae. Tous deux sont des Goniothorax.

\section{Leptothorax (Goniothorax) Grisoni n. sp.}

భ. Longueur: $2^{\mathrm{mm}}, 7-2^{\mathrm{mm}}, 9$. Mandibules étroites, densément striées, assez luisantes, armées de 4 dents. Epistome fortement lobé, entier devant comme chez le précédent. Aire frontale distincte, un peu arrondie derrière. Tête presque $1 \frac{1}{3} 3$ fois plus longue que large, ayant presque exactement la même forme que chez l'Evelynae. Mais les angles postérieurs sont presque aigus et l'occiput subbordé entre sa face antérieure et sa face postérieure. Les yeux, situés au milieu, assez grands, paraissent légèrement enfoncés entre deux convexités des còtés. Le scape atteint le bord postérieur. Antennes de 12 articles. Le dernier article du funicule n'est pas 2 fois plus long que le précédent; les articles 3 à 7 ne sont qu'un peu plus épais que longs; tous les autres plus longs qu'épais. Thorax très peu convexe en tout sens, subdéprimé et subbordé latéralement. Angles antérieurs du pronotum très distincts, subdentiformes. Suture promésonotale assez distincte, aussi distincte que la suture mésoépinotale. Aucune échancrure mésoépinotale, pas même d'enfoncement à la suture. Epines de l'épinotum obtuses, longues comme les ${ }^{2} / 3$ de leur intervalle. Face basale presque 2 fois plus longue 
que large; face déclive bien plus courte. Nœud du pétiole cubique, à peine plus large que long, presque sans pédicule antérieur, rappelant celui du Leptothorax corticalis Schenk. Postpétiole très petit, presque de moitié plus court que le nœud du pétiole, mais au moins 2 fois plus large que long, bien plus large que le nœud du pétiole. Abdomen tronqué devant. Cuisses renflées au milieu.

Mat, grossièrement réticulé. Sur la tête, les réticulations sont un peu séparées les unes des autres sous formes de fossettes, rappelant un peu la sculpture de l'Evelynae; leur fond est raboteux. Abdomen lisse et luisant, de même que les membres et la face déclive de l'épinotum. Les réticulations ont un aspect légèrement longitudinal sur le front et transversal sur la face basale de l'épinotum. Pilosité épaisse, très courte (à peine plus longue que chez l'innocens), tronquée, jaunâtre, médiocrement abondante sur le corps, nulle sur les membres. Pubescence diluée partout et très adjacente. D'un brun presque noir, avec les scapes, la première moitié du funicule, les mandibules, les tarses et les articulations d'un jaunâtre sale. Le tiers postérieur de l'abdomen d'un brun clair.

$\sigma^{\Upsilon}$ Longueur : $3^{\mathrm{mm}}$. Tète rectangulaire arrondie, à peine plus longue que large. Le scape dépasse un peu le bord postérieur; antennes de 13 articles assez égaux, sauf le dernier. Thorax plus large que la tête; épinotum inerme, arrondi, sa face déclive courte. Nœud du pétiole plus long que large presque sans pédicule antérieur, ailes subhyalines, à nervures et tache pâles. Noir, mandibules et membres d'un jaune pâle. Les mandibules ont deux dents.

St-Gabriel, Congo (KонL), sous l'écorce d'un Mango. Cette espèce rappelle un peu le genre Nesomyrmex Wheeler, mais elle a 12 articles aux antennes.

M. Emery croit pouvoir identifier mon sous-genre Caulomyrma avec le genre Nesomyrmex Wheeler, fondé sur une seule q. Mais le genre de Wheeler a la tête bordée derrière. Pour le moment. je crois devoir maintenir mon sous-genre Caulomyrma jusqu'à ce que la question soit mieux éclaircie. 
Cataulacus Weissi Sant.

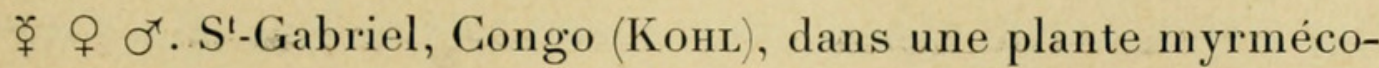
phile (Randia myrmecophila de WiLde).

우 (encore inédite). Longueur: $4^{\mathrm{mm}}, 5$. Du reste toute semblable à l'ouvrière, mais l'abdomen est presque 2 fois plus long que large, avec les còtés presque parallèles. Mésonotum et scutellum régulièrement ridés en long, un peu plus étroits que la tête. Ailes jaunâtres, à nervures pâles. Du reste comme l'ouvrière.

$\sigma^{x}$ Longueur : $3^{m m}, 5$. Tête presque triangulaire, très rétrécie devant, aussi large derrière que le thorax, avec 2 dents pointues de chaque còté. Epines de l'épinotum longues et grêles, aussi longues que leur intervalle. Un appendice bidenté sous le pétiole. Du reste comme la 오.

Cataulacus brevisetosus For. r. Lujae For.

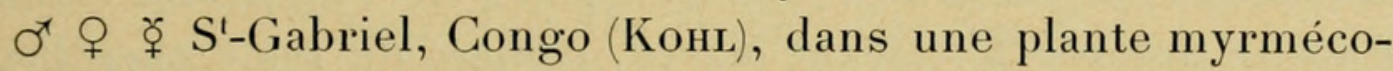
phile. Il s'agit d'une variété plus petite encore que le type. భ. Longueur: $2^{\mathrm{mm}}, 5-2^{\mathrm{mm}}, 6$. ๆ. Longueur: $3^{\mathrm{mm}}, 9-4^{\mathrm{mm}}$. $\sigma^{x}$. Longueur: $3^{\mathrm{mm}}, 8$.

Cataulacus guineensis $\mathrm{Sm}$.

¡ $q$ Motombé sur l'Okiavo. Dans un nid de Guêpes maçonnes abandonné, sur un tronc d'arbre. St-Gabriel, courant en para- biose amicale avec un Cremastogaster. Congo (KoнL).

Cataulacus erinaceus Stitz.

§ q Congo (КонL).

우 (encore inédite). Longueur: $9^{m m}, 5$. Thorax aussi large que la tête. Mésonotum, scutellum et face basale de l'épinotum avec des rides longitudinales régulières. Abdomen seulement un peu plus long que chez l'ouvrière; les ailes manquent.

Strumigenys (Trichoscapa) Escherichi For. r. cognata Sant. Congo (КонL).

Strumigenys (Trichoscapa) Maynei n. sp.

భ. Longueur: $1^{\mathrm{mm}}, 9-2^{\mathrm{mm}}$. Diffère de l'Escherichi For. et de 
sa r. cognata par le pronotum plus large, sans carène médiane, très distinctement et assez densément ridé en long. Il n'y a pas d'escalier derrière le mésonotum, par contre une forte échancrure mésoépinotale, le promésonotum étant fort convexe et la face basale de l'épinotum aussi, sur le profil. Tout l'Insecte est mat, sauf l'abdomen, le postpétiole et les membres. D'un roussâtre franc, parfois d'un roux jaunâtre. La pubescence est bien plus claviforme que chez Escherichi et sa race cognata Sant. (partout). Ressemble aussi un peu à la biconvexa Sant., mais bien plus grande, n'ayant pas de membrane à l'épinotum, puis une autre sculpture. Pour le reste, comme Escherichi.

q. Longueur: $2^{\mathrm{mm}}, 5$. Thorax un peu plus large que la tête. Mésonotum assez fortement ridé et réticulé, plus faiblement à son milieu, devant. Une tache brune entre les ocelles. Les ${ }^{2} / 3$ antérieurs de l'abdomen bruns. Epines de l'épinotum fortes. Pilosité dressée fortement clavée, comme du reste chez l'ouvrière, et un peu plus courte que chez Escherichi. Les ailes un peu enfumées de jaune brunâtre. Pubescence un peu moins clavée que chez l'ouvrière.

$\sigma^{x}$. Longueur: $2^{\mathrm{mm}}, 2$. Une petite lacune entre la base des mandibules. Antennes longues et assez filiformes. Scape à peine 2 fois plus long qu'épais. Tête bien plus longue que large; thorax plus large que la tête. Une petite dent triangulaire entre les faces de l'épinotum dont la déclive est plane et oblique. Nœud du pétiole arrondi, plus court que son pédicule antérieur. Abdomen lisse; tout le reste du corps mat, densément réticuléponctıé. Ailes d'un jaune pâle à nervures pâles, d'un brun noir avec les membres, les mandibules et le tiers postérieur de l'abdomen roussâtres.

Congo (КонL).

Une variété récoltée par M. R. Mayné au Congo ( $\not$ et 우) se distingue par sa tête un peu plus courte et plus large, et surtout par sa pubescence encore plus clavée. Les stries du promésonotum sont légèrement plus fines, je l'appelle var. latiuscula n. var. La taille est la même. 
Acromyrmex Mölleri For. r. Meinerti For. v. globoculis $\mathrm{n}$. var.

ఢ. Longueur: $4^{\mathrm{mm}}, 5-5^{\mathrm{mm}}, 2$. Se distingue du type de la race par ses yeux plus convexes, hémisphériques. Les épines supérieures de l'occiput sont aussi plus courbées, la tête un peu plus étroite derrière, la pubescence un peu plus forte et la couleur un peu plus terne. Ressemble à la r. modesta For., à laquelle elle fait transition, mais qui a les yeux bien moins convexes.

Guyane anglaise, reçu par M. Crawley.

L'ouvrière de Meinerti est longue de 3 à $5^{\mathrm{mm}}$. Elle est bien moins grêle et bien moins pubescente que la modesta. Sa tête est bien plus large, moins rétrécie derrière où elle a des épines obliques et presque droites (horizontales et courbées chez la modesta). Je l'ai reçue des provinces de Rio-de-Janeiro, de São Paulo, de Para, etc.

\section{$4^{\text {me }}$ Sous-famille Dolichoderinae For.}

Semonius Schultzei For.

§ St-Gabriel, Congo (КонL).

\section{Tapinoma Arnoldi For.}

భ (?) (encore inédite). Longueur: $2^{\mathrm{mm}}, 5$. Tête rectangulaire, bien plus longue que large, à bord postérieur droit; plus large que le thorax. Le scape n'atteint que le cinquième postérieur de la tête. Articles 2 à 10 du funicule plus épais que longs. Yeux au tiers antérieur. Thorax allongé, subcylindrique. Pilosité et pubescence comme chez l'ouvrière. Entièrement brune. Les membres comme le reste.

Natal, récoltée autrefois par M. Haviland. Je rapporte cette o à l'Arnoldi avec un certain doute.

Engramma Kohli $\mathrm{n}$. sp.

․ Longueur: $1^{\mathrm{mm}}, 8-2^{\mathrm{mm}}, 2$. Mandibules luisantes, fortes, à bord externe fortement courbé, armées d'au moins 7 ou 8 dents, poilues, peu et très finement ponctuées. La forte échancrure de l'épistome occupe le $5^{\text {me }}$ de son bord antérieur; elle est semi-

Rev. Suisse de Zool. T. 24. 1916. 
circulaire, aussi profonde que large devant. Milieu de l'épistome presque deux fois plus haut que l'échancrure. La tète est plus ou moins trapéziforme, presque 2 fois plus large derrière qu'à son bord antérieur, un peu moins profondément échancrée derrière que chez E. Lujae For., avec les còtés beaucoup moins convexes; elle est aussi longue que sa largeur postérieure. Les petits yeux, situés au milieu, n'ont guère que 10 à 12 facettes. Le scape atteint le bord postérieur de l'occiput. Articles 2 à 9 du funicule plutòt un peu plus épais que longs. Promésonotum faiblement convexe. Echancrure mésoépinotale profonde et assez large. Epinotum fort convexe; sa face basale presque double de la déclive. Ecaille et abdomen comme chez E. Lujae.

Entièrement luisante et lisse. Ponctuation très fine et très éparse. Pilosité dressée pâle, très éparse sur le corps, nulle sur les membres. Pubescence jaune, fort distincte partout, très adjacente, formant un faible duvet qui ne cache nullement la sculpture. D'un jaune assez terne, un peu roussâtre. Abdomen brun.

․ Longueur: $4^{\mathrm{mm}}, 7-6^{\mathrm{mm}}$. Mandibules comme chez l'ouvrière, mais armées de 9 à 10 dents. Bord postérieur de la tête plus faiblement échancré. Le scape n'atteint pas le bord postérieur ; il s'en faut de son épaisseur. Tête du reste comme chez l'ouvrière, aussi large derrière que le thorax; celui-ci assez plat en dessus, y compris la face basale de l'épistome (convexe chez l'ouvrière): face déclive oblique, 2 fois plus longue. L'écaille est marquée devant par un bourrelet convexe; le pétiole a en dessous une lamelle allongée, longitudinale, pâle, à demi translucide, qui est indistincte chez l'ouvrière. Abdomen grand.

Tout le corps assez densément et beaucoup plus fortement ponctué que chez l'ouvrière, ce qui le rend subopaque. Membres moins densément ponctués. Pubescence et pilosité comme chez l'ouvrière; cette dernière un peu plus abondante. Brune. Tête et thorax variés de roussâtres. Mandibules, antennes et cuisses roussâtres; pédicule, tibias et tarses d'un jaune sale.

St-Gabriel, Congo (КонL). Pris sous 4 numéros différents dans des plantes myrmécophiles. 
Engramma Zimmeri For. r. okiavoensis n. st.

Longueur: $4^{\mathrm{mm}}-4^{\mathrm{mm}}, 4$. Diffère du type par sa taille plus grande, par les còtés de la tête moins convexes, par son scape bien plus court ne dépassant le bord postérieur que de moins d' $1 / 5$ de leur longueur. Les arêtes frontales sont encore plus distantes l'une de l'autre que chez le type. L'épinotum est plus long, bien plus long que large. Les deux stigmates de la large échancrure thoracique proéminent au fond du métanotum. L'orifice du cloaque est presque apical, comme chez les Technomyrmex. La couleur est inverse de celle du type: noire, avec l'abdomen, les funicules, les mandibules et le bout des tarses d'un roux jaunàtre. Parfois l'abdomen est brun.

Sur le fleuve Okiavo, près de St-Gabriel, Congo (KoHL), dans un grand nid d'un blanc gris, mou, tissé et mêlé de matières végétales fines, adossé à un tronc d'arbre. Le nid avait $50 \mathrm{~cm}$. de long, 20 à $30 \mathrm{~cm}$. de large et 3 à $4 \mathrm{~cm}$. d'épaisseur. La partie de l'écorce recouverte par le nid était remplie de grands Coccides cultivés par les Fourmis. Celles-ci sont paisibles et répandent une odeur particulière quand on les touche. Cette odeur ressemble à celle des machines.

Comme pour le type, c'est avec doute que je rattache cette race au genre Engramma.

Engramma Ilgii For.

ґ. Makanga, Congo (Конц) sur des nectaires floraux, cultivant aussi des Coccides. Exemplaires un peu plus petits que le type. Longueur : $2^{\mathrm{mm}}, 5-2^{\mathrm{mm}}, 6$.

Engramma Laurenti Em. v. congolensis n. var.

¡ Longueur: $2^{\mathrm{mm}}, 4-2^{\mathrm{mm}}, 8$. Diffère du type par sa couleur beaucoup plus claire, entièrement d'un jaune roussâtre, terne et pâle chez tous les individus. La tète est aussi légèrement moins large, surtout derrière; le promésonotum est un peu moins convexe et la face basale de l'épinotum un peu plus haute. La pilosité et la pubescence sont un peu plus faibles, la ponctuation par contre plus forte, surtout sur la tête, qui est subopaque devant, réticulée-ponctuée. 
\& (?) Longueur : $5^{\mathrm{mm}}, 4$. D'un brun noirâtre. La large et profonde échancrure de l'épistome est comme chez le type de l'espèce et de la race. Subopaque, densément ponctuée, en partie réticulée-ponctuée. Membres et mandibules d'un brun plus roussâtre. Les ailes manquent.

St-Gabriel et Bengemeza, Congo (KoHL), dans des plantes myrmécophiles, comme l'Engramma Kohli. Je ne crois pas faire erreur en rattachant la femelle prise isolément à cette même race.

Engramma Lujae For. r. Wasmanni n. st.

گ. Longueur: $2^{\mathrm{mm}}, 7-3^{\mathrm{mm}}$. Même taille que le type de l'espèce dont elle diffère surtout par sa sculpture beaucoup plus forte, densément ponctuée, qui la rend subopaque, du moins en partie, et par sa pubescence beaucoup plus forte aussi. De plus, la tête est moins large, moins profondément échancrée derrière, avec les còtés moins convexes. Les yeux sont notablement plus petits. La couleur est d'un brun noirâtre, avec la tête d'un roux jaunâtre, à peu près comme chez le type.

․ Longueur: $2^{\mathrm{mm}}, 7$. Ailes brunâtres avec une cellule discoïdale distincte, une seule cellule cubitale et la nervure transverse s'unissant à la nervure cubitale à son point de partage. La tête est indistinctement plus longue que large avec les còtés très peu convexes et le bord postérieur superficiellement concave. La tête est bien moins large que celle de l'ouvrière, aussi large que le thorax qui est petit et assez plat. Couleur brune avec l'abdomen presque noir ; le front, les joues et les mandibules d'un roux un peu brunâtre. Tout le reste comme chez l'ouvrière, en particulier l'épistome.

๙. Longueur: $2^{\mathrm{mm}}, 9$. Un peu moins luisant que le type de l'espèce; couleur un peu plus foncée, presque noire; du reste identique.

Congo (KоHL); reçue par M. Wasmann.

Engramma Wolfi n. sp. (Fig. 1.)

భ. Longueur: $3^{\mathrm{mm}}, 8-4^{\mathrm{mm}}, 1$. Longue et étroite, plus encore que l'E. Zimmeri. Bord terminal des mandibules très long, armé 
d'au moins dix dents, bien plus long que le bord interne. Echancrure de l'épistome très large, au moins comme un tiers du bord antérieur, néanmoins semi-circulaire, presque aussi profonde que sa largeur, suivie derrière, au milieu, d'une impression triangulaire, toute analogue à l'aire frontale. Le milieu de l'épistome, comme chez l'E. Lujae, est aussi long de còté qu'au milieu jusqu'à l'échancrure. Aire frontale indistincte. Tète ovale, à còtés très convexes, d'un bon cinquième plus longue qu'elle est large au tiers postérieur, très rétrécie devant et derrière, où ses angles sont très arrondis et où le milieu est concave, le bord postérieur étant indistinct. Yeux très grands, situés plutòt en arrière du milieu, peu convexes, occupant le quart des côtés. Le scape

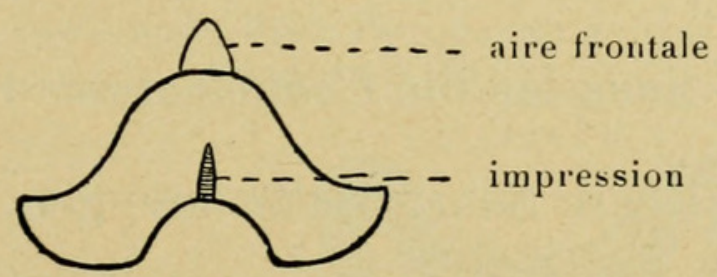

FIG. 1.

Epistome de l'Engramma Wolfi For. $\Varangle+$. (Emma Forel, del.)

dépasse l'occiput des deux cinquièmes de sa longueur. Tous les articles du funicule, qui n'est nullement renflé à l'extrémité, subégaux, presque deux fois plus longs qu'épais. Thorax étroit, allongé; pronotum un peu plus large que long. Suture promésonotale enfoncée, séparant la convexité du mésonotum, qui est plus long que large, de celle du pronotum. Echancrure mésoépinotale forte et surtout très large. Au fond, un métanotum fort distinct, avec ses stigmates. Vu de dessus, l'épinotum, encore plus étroit que le mésonotum, est $1 \frac{1}{2} 2$ fois plus long que large ; vu de profil, il est bossu, avec les deux faces convexes, mais surtout le sommet. Face déclive un peu plus longue que la basale. Pétiole allongé et étroit, ayant à peine un bord d'écaille un peu aigu devant, entièrement recouvert par l'abdomen qui forme en avant une pointe obtuse avancée. Pattes très longues.

Thorax, sauf la face déclive de l'épinotum, fortement réticulé- 
ponctué et presque mat (chez certains individus seulement densément ponctué et subopaque). Tète densément ponctuée, subopaque; abdomen luisant, faiblement chagriné, avec direction transversale des rides. Membres ponctués. Pilosité dressée presque nulle, sauf aux deux extrémités du corps et en dessous. Pubescence assez longue et assez abondante sur le corps et sur les membres, sans toutefois cacher la sculpture. Presque noire. Mandibules et membres bruns. Tarses et articulations plus clairs, un peu jaunâtres.

S'-Gabriel, Congo (KонL), dans la forêt vierge sur le sol, parmi des feuilles pourries; prise une fois à la lampe. Cette espèce est très caractéristique, fort différente de Zimmeri et des autres. Reçue aussi par M. Wasmann.

$5^{\text {me }}$ Sous-famille Camponotinae For.

Genre Santschiella nov. gen.

$\Varangle$. Antennes de 12 articles. Ouverture du cloaque apicale, circulaire, ciliée. Mandibules triangulaires de forme ordinaire. Epistome triangulaire, arrondi derrière et prolongé entre les insertions des antennes. Arêtes frontales presque nulles, limitées au bord intérieur de l'insertion des antennes. Yeux énormes, même plus grands que chez les Gigantiops; ils convergent d'arrière en avant où ils se rapprochent fortement l'un de l'autre. Trois ocelles frontaux. Tête bordée en dessous, derrière, d'une arête transparente, terminée par un bord abrupt et suivie d'une dent. Thorax profondément échancré entre le mésonotum et l'épinotum. Ce dernier bossu, armé de deux épines à sa face déclive. Pétiole surmonté d'un nœud très épais, qui est tronqué et bordé derrière. Gésier inconnu.

Je place ce genre dans ma section des Procamponotinae à côté des Myrmoteras et des Dimorphomyrmex. Je suis, en effet, obligé de réunir le genre Dimorphomyrmex aux Procamponotinae, eu égard à son gésier qui ressemble beaucoup à celui des Myrmoteras, d'après la figure d'André. Je donne ici la figure 
du gésier du Genre Myrmoteras et l'on pourra juger (fig. 4). Je n'ai pas osé disséquer le gésier du nouveau genre Santschiella, représenté seulement par deux exemplaires dont je dois rendre l'un. Ce singulier genre de Camponotinae a des affinités diverses, d'un còté avec les Dolichoderinae (épistome), de l'autre avec Myrmoteras (arètes frontales), mème avec les Ponerinae (pétiole). Il a tout l'aspect fossile d'un reliquat phylogénique.

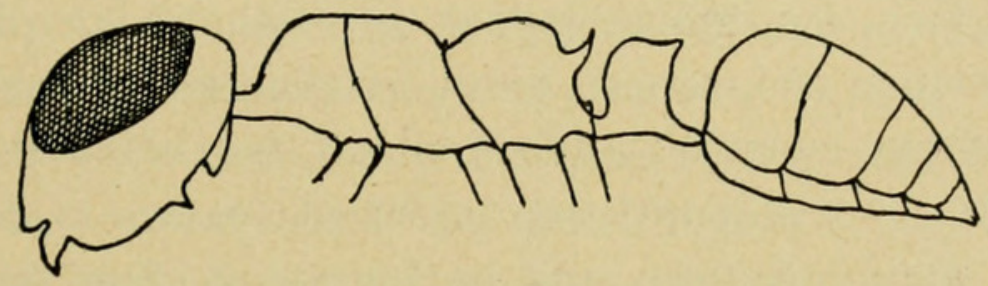

FIG. 2.

Santschiella Kohli For. $\Varangle$, vue de côté.

(Emma Forel, del.)

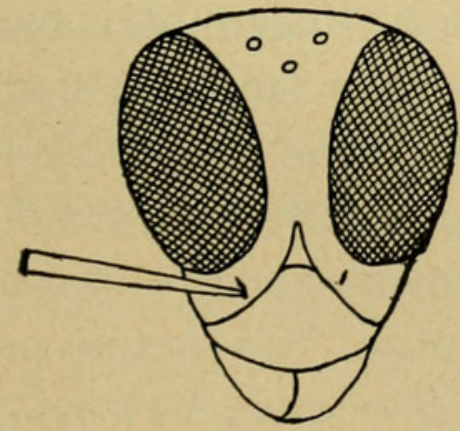

FIG. 3.

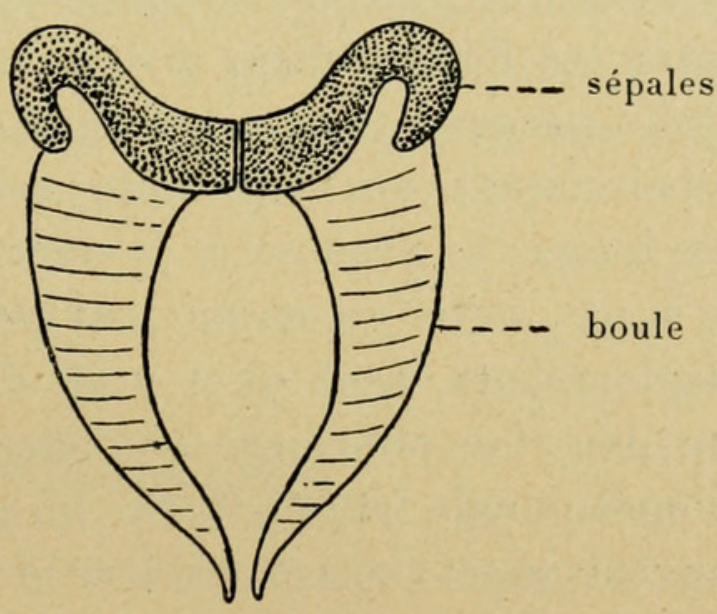

Fig. 4 .

Santschiella Kohli For. $\Varangle, \quad$ Gésier du Myrmoteras Binghami For. tête vue de devant.

(Emma Forel, del.)

(Emma Forel, del.)

On pourra fixer définitivement sa position en étudiant plus tard son gésier, quand on retrouvera la Fourmi. En attendant je renvoie aux deux figures 2 et 3 .

Santschiella Kohli n. sp. (Fig. 2 et 3.)

$\Varangle$. Longueur: $3^{\mathrm{mm}}, 6$. Mandibules luisantes, lisses, avec quelques points épars très fins, armées de 7 ou 8 dents irrégulières, 
dont une ou deux très petites; leur bord extérieur est plus long que le bord terminal. Le bord antérieur de l'épistome est régulièrement convexe. Epistome sans carène, avec une fossette clypéale se confondant avec la fossette antennaire. Aire frontale grande, plus haute que sa largeur; sillon frontal indistinct, très faible. Vue de devant (fig. 3), la tête paraît un peu plus longue que large, fortement rétrécie devant, avec le bord postérieur faiblement convexe et trois ocelles fort distincts, mais assez. petits. De face, les énormes yeux paraissent bien plus larges que la distance qui sépare leurs extrémités antérieures. Ils sont fortement convexes, surplombant les còtés de la tête. Vue de còté, la tête paraît extrêmement épaisse et courte, pas beaucoup moins épaisse que sa longueur. Son tiers postérieur est bordé d'une arête translucide qui s'élève d'arrière en avant, où elle se termine par un bord abrupt. Un peu plus en avant, on voit une seconde dent triangulaire sur le profil. Entre les deux arètes, la tête est un peu excavée. Le scape atteint presque le $1 / 4$ postérieur de la tête. Le funicule va en s'épaississant vers l'extrémité; ses articles 2 à 10 sont plus épais que longs; le premier et le dernier seuls plus longs qu'épais. Le scape, retiré en arrière, est entièrement caché sous les yeux surplombants, sans qu'il y ait de scrobe. Pronotum, sans le cou, deux fois plus large que long et fortement convexe. Suture promésonotale très distincte, un peı enfoncée. Mésonotum peu convexe, mais abaissé derrière, devant l'échancrure. Au fond de cette dernière, apparaissent les deux stigmates métanotaux. Face basale de l'épinotum fortement convexe (bossue); la face déclive aussi longue que la basale, ayant au milieu de ses còtés une forte épine à large base, pointue à l'extrémité et recourbée en haut. Tout à la base, deux très petites dents obtuses subépinotales. Le nœud du pétiole est un peu plus haut qu'épais et tronqué devant et derrière, $1 \frac{1}{3}$, fois plus large que long. Mais tandis que sa face antérieure s'arrondit en haut pour passer au sommet, qui est convexe, la troncature postérieure est concave et bordée, terminée de chaque còté, en haut, par une dent triangulaire obtuse de chaque còté du sommet. Le bord supérieur 
du nœud est lui-mème relevé. L'abdomen est plutòt petit, de forme ordinaire. Les cuisses antérieures sont un peu renflées.

Médiocrement luisante, sauf l'occiput et les pattes qui sont lisses et fort luisants. Tout le reste est extrêmement finement pointillé ou chagriné et légèrement subopaque. Sur le devant de la tète, qui devient presque mat, cette sculplure est plus profonde et plus dense, prenant une direction longitudinale. Pubescence très courte et fort éparse partout. Pilosité dressée nulle, sauf vers l'extrémité de l'abdemen, surtout dessous, où elle est jaunâlre, courte, obtuse et tronquée au bout.

D'un roussâtre clair, avec les pattes, les mandibules, les scapes et le premier article du funicule d'un jaune roussâtre. Le reste du funicule et une bande transversale assez étroite à l'extrémité de chacun des deux premiers segments de l'abdomen d'un brun foncé.

St-Gabriel, Congo (Конц). Je renvoie aux figures 2 et 3 . Cette espèce a été prise avec d'autres. Il n'y a pas d'indication sur ses mœurs. Je suppose qu'elle vit sur les arbres, d'où elle était tombée ou descendue.

Plagiolepis exigua For.

‘ Congo (KонL).

Plagiolepis pygmaea Latr. v. punctum For.

¡ St-Gabriel, Congo (KонL).

Plagiolepis pygmaea Latr. v. mediorufa n. var.

广. Longueur: $1^{\mathrm{mm}}, 1-1^{\mathrm{mm}}, 4$. Diffère du type de l'espèce par sa taille, et par sa tête plus étroite, qui n'est nullement échancrée, plutòt convexe derrière, avec les còtés convexes. La couleur est caractéristique. Tête et abdomen bruns. Thorax d'un jaune plus ou moins rougeâtre. Cuisses et funicules brunâtres, le reste des membres jaunâtre.

St-Gabriel, Congo (КонL) dans une plante myrmécophile (3 numéros). Cette variété ressemble à s'y méprendre à la $P l$. (Anacantholepis) Van der Kelleni For. v. polita Sant., mais avec le métanotum d'une vraie Plagiolepis. 
Acantholepis capensis Mayr. v. anceps $\mathbf{n}$. var.

ఢ. Longueur: $2^{\mathrm{mm}}, 4-2^{\mathrm{mm}}, 7$. La pilosité est semblable à celle de la race depilis Em. L'écaille et l'épinotum tendent un peu à la forme de l'A. simplex For. Du reste semblable au type.

Congo (КонL), nichant dans la terre.

Acantholepis capensis Mayr. r. Junodi n. st.

ఢ. Longueur: $2^{\mathrm{mm}}, 5-2^{\mathrm{mm}}, 8$. Diffère de la r. incisa For., dont elle a l'écaille bispineuse, par ses pattes et ses scapes, couverts de soies blanchâtres subadjacentes, par les soies jaunâtres et assez pointues du corps, par sa tète beaucoup plus large, par ses scapes plus courts, ne dépassant guère la tête que du quart, enfin par l'incisure moins profonde du thorax. Elle diffère de curta Em. par les épines de l'écaille et par sa tête moins large.

Shilowana, Transvaal (Junod). Reçue du Musée de Genève.

Acantholepis simplex For. v. minuta n. var.

ఢे. Longueur: $1^{\mathrm{mm}}, 5$. Diffère du type, outre sa très petite taille, par sa stature plus robuste et par ses scapes plus courts qui ne dépassent guère le bord postérieur de plus de leur épaisseur. Elle diffère de la v. laevis Sant. en outre par sa couleur noir de jais et par sa tête plus large.

Shilowana, Transvaal (Junod). Reçue du Musée de Genève.

Prenolepis Kohli n. sp.

ґ. Longueur: $3^{\mathrm{mm}}, 6--4^{\mathrm{mm}}$. Mandibules presque mates, très densément striées, armées de 6 dents. Epistome caréné, fortement vouté, surtout au quart antérieur et au quart postérieur de la carène. Aire frontale triangulaire; arêtes frontales presque droites, à peine divergentes; sillon frontal distinct. Yeux au tiers postérieur, grands. Tête presque d'un quart plus longue que large, à còtés assez convexes, du reste rectangulaire, aussi large devant que derrière, à bord postérieur droit (à peine faiblement concave au milieu et convexe de côté). Le scape dépasse le bord postérieur de la moitié de sa longueur. Articles du funicule 3 fois plus longs que larges, le premier plus court. Promésonotum faiblement convexe. Mésonotum au moins $1 \frac{1}{2}$ fois 
plus long que large. Echancrure thoracique assez forte et large. Epinotum bossu; sa face basale fort convexe; sa face déclive oblique, presque plane, plus longue que la basale. Les stigmates métanotaux proéminent au fond de la suture. Ecaille très haute, verticale, assez étroite, obtuse au sommet, un peu convexe devant, plane derrière. Pattes longues.

Mate ou à peine subopaque. Densément réticulée-ponctuée; abdomen plutòt subopaque. De gros points enfoncés ou fossettes un peu allongées sur l'abdomen et l'occiput. Des soies assez grossières, mais pointues, médiocrement abondantes sur l'abdomen et la tête, plus rares sur le thorax, nulles sur les membres. Pubescence diluée partout et courte, entièrement adjacente. D'un roux brunâtre; pattes et antennes d'un roux jaunâtre.

ㅇ (?) Longueur: $5^{\mathrm{mm}}-5^{\mathrm{mm}}, 2$. Tête bien plus étroite que le thorax, à bord postérieur droit et large. La tète est un peu plus large que longue, dépassée par le scape d'un bon tiers de sa longueur. Le second article du funicule est plus court que les autres, qui ne sont guère plus de 2 fois plus longs que larges. Ailes faiblement enfumées de jaune brunâtre, à nervures brunes. Pilosité plus rare et plus courte que chez l'ouvrière; écaille moins obtuse au sommet. Brune. Antennes, mandibules et tibias roussâtres. Sculpture, pubescence et tout le reste comme chez l'ouvrière.

S'-Gabriel, Congo (Конц). C'est avec doute que je rapporte la $q$ à l'ouvrière; elle a un autre numéro, et les différences du funicule et de l'écaille donnent à réfléchir. Celte espèce, malgré son thorax peu rétréci, rentre, comme première espèce africaine, dans le genre Prenolepis s. str., qui se trouve ainsi réparti dans les cinq parties du monde, même dans les faunes néarctique et néotropique.

\section{Prenolepis (Nylanderia) Butteli For. r. Bryanti n. st.}

§. Longueur: $2^{\mathrm{mm}}$. Aussi petite que le type de l'espèce dont elle diffëre surtout par son éclat métallique pourpré. La tête est en outre bien plus étroite derrière, avec les còtés plus con- 
A. FOREL

vexes. Le scape est un peu plús court et la sculpture plus forte (tête et thorax presque mats). A part l'éclat métallique, la couleur est plus brune. Les tarses et les tibias antérieurs et moyens sont blanchâtres.

Mont Matang, Ouest Sarawak (G. E. Bryant) envoyée par M. Donisthorpe.

Prenolepis (Nylanderia) Grisoni n. sp. (Fig. 5.)

$\underset{+}{\gamma}$. Longueur : $2^{\mathrm{mm}}-2^{\mathrm{mm}}, 1$. Mandibules luisantes, ponctuées, fortement croisées, étroites, à bord terminal fort oblique. Epistome subcaréné, convexe. Tète d'un bon quart plus longue que large, presque plus large devant que derrière, où son bord postérieur est assez convexe, tandis que les còtés le sont à peine. Yeux au milieu, assez grands et plats. Le scape dépasse la tête

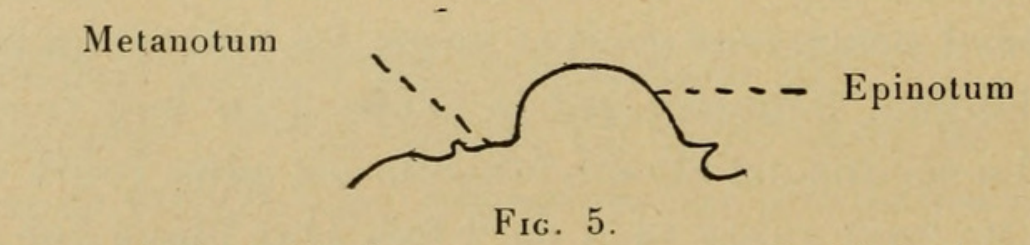

Prenolepis (Nylanderia) Grisoni For. $\Varangle$ Profil du thorax.

(Emma Forel, del.)

de plus des ${ }^{2} / 5$ de sa longueur. Promésonotum assez faiblement convexe. Mésonotum presque $1 \frac{1}{2} 2$ fois plus long que large. Un long métanotum, aussi long que les ${ }^{3 / 4}$ du mésonotum, avec 2 stigmates proéminant en dents au milieu, constituent une constriction notable du thorax. L'échancrure de ce dernier est située entre le métanotum et l'épinotum. Ce dernier forme une bosse très convexe, presque hémisphérique, bien plus haute que le reste du thorax, sans distinction entre face basale et déclive, mais terminée derrière, en bas, de chaque còté, par une petite dent triangulaire, pointue, très nette. Ecaille assez épaisse et fort basse, inclinée en avant, obtuse au sommet, Pattes plutòt longues. Premier segment de l'abdomen avancé devant.

Tout le corps lisse et luisant avec des aspérités sétigères et, par-ci par-là, un pli longitudinal. Corps et membres parsemés 
des soies raides, obtuses, brunes et épaisses propres aux Nylanderia, plus courtes sur les membres que sur le corps. Pubescence très diluée. Abdomen, écaille et épinotum d'un brun très foncé. Tête, cuisses, tibias, scapes et pronotum d'un brun plus clair. Mésonotum, mandibules, funicules, articulations et pattes d'un jaune plus ou moins brunàtre.

Bengemeza, Congo (КонL) dans une plante myrmécophile. Cette singulière espèce, bien caractérisée par la forme de la tête, de l'épinotum et du métanotum allongé, est absolument différente de Traegaordhi, albipes, Jaegerskiöldi et Weissi, mais il n'est pas impossible qu'elle soit l'ouvrière encore inconnue de Waelbroecki Em., dont je ne connais que le mâle.

Prenolepis (Nylanderia) longicornis Latr. v. Hagemanni For. ¡ S'-Gabriel, Congo (KoHL), sous une tuile.

Machaeromyrma nov. subgen. Je me permets d'instituer pour le Cataglyphis bombycina Rog. le nouveau sous-genre Machaeromyrma, caractérisé par son grand dimorphisme et par la singulière structure en épée des mandibules du 2 . Le nid et les mœurs de cette espèce sont aussi fort caractéristiques et différents des autres.

Camponotus (Dinomyrmex) Wellmanni For.

$\Varangle$ major (encore inédite). Longueur : $17^{\mathrm{mm}}$. Tête large de $6^{\mathrm{mm}}$ et longue de $6^{\text {mn }}, 5$ (sans les mandibules). Elle est en forme de trapèze, élargie d'avant en arrière, profondément et largement échancrée à son bord postérieur. Angles occipitaux plutôt étroits, à peine dépassés par le scape obliquement placé. La tête est subopaque, plutòt mate, densément réticulée ou réticuléeponctuée. Epistome caréné, avec un lobe antérieur rectangulaire. Tout le reste comme chez la $ᄋ$ et l'ouvrière minor

Bamayanga, Congo, Musée du Congo belge. Reçu de M. SchouTEDEN.

Camponotus (Dinomyrmex)Wellmanni For.v.rufipartis n.var. $\Varangle$ minor. Longueur : $13^{\mathrm{mm}}$. Tête plus longue et surtout 
relativement plus étroite devant que chez le type, plus d'une fois et demie plus longue que sa largeur antérieure ; les còtés, assez convexes, divergent bien moins en avant. Le scape ne dépasse le bord postérieur de guère plus que de sa moitié. Le thorax, le funicule et les tarses sont d'un brun roussâtre, en partie roux; le reste est d'un brun foncé moins noir que chez le type.

ㅇ․ Longueur: environ $23^{\mathrm{mm}}$. Tète à peine plus longue, mais un peu plus étroile que chez le type. Couleur comme chez l'ouvrière minor, mais les pattes entières, y compris les hanches, et une partie des métapleures sont roussâtres. La pubescence des pattes est un peu plus soulevée que chez le type.

$\sigma^{x}$. Longueur: $12^{\mathrm{mm}}, 8$. Mandibules longues à bord terminal tranchant. Epistome comme chez la $q$. Tête $1 / 2$ fois plus longue que large, à bord postérieur confondu avec le bord articulaire, mais sans cou. Les còtés sont droits et parallèles devant les yeux, convexes et convergents derrière. Le scape dépasse le bord postérieur de ses deux tiers. Ailes teintes d'un jaune un peu roussâtre. D'assez grosses fossettes sur le mésonotum. Brun; tibias, tarses, funicules, mandibules et quelques taches sur le thorax derrière roussâtres.

Congo (KонL).

Camponotus (Myrmoturba) maculatus F. r. Solon For. ఢे. Lumbulumbu, Congo (KонL), dans un arbre creux.

Camponotus (Myrmoturba) maculatus F. r. Brutus For.

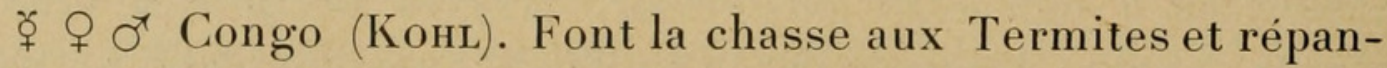
dent une odeur âcre.

Camponotus (Myrmoturba) acwapimensis Mayr.

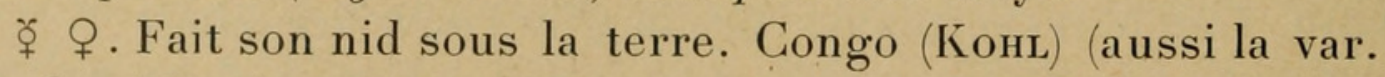
Poultoni For.).

Camponotus (Myrmoturba) conspicuus Sm. r. Williamsi n. st.

$\Varangle$ major. Longueur: $10^{\mathrm{mm}}, 5-13^{\mathrm{mm}}$. Diffère du type par ses scapes plus longs, dépassant le bord postérieur de plus d'un 
tiers de leur longueur, par sa stature plus grèle, par sa tête plus étroite derrière, par son écaille plus épaisse vers la base, par les poils plus longs sur le corps et plus abondants sur les joues, enfin par sa couleur plus foncée, d'un brun châtain uniforme, avec les hanches et les cuisses d'un roux jaunâtre. Il diffère en outre du fumidus Rog. v. festinatus Wh. par ses poils obliques aux tibias, par ses arêtes frontales plus divergentes, par son épistome plus large devant, par des fossettes sur les joues et par son écaille plus épaisse, presque aussi épaisse à la base que haute.

Guadeloupe (Antilles), sur des arbres autour du tronc desquels il construit des tunnels à l'aide de détritus. Récolté par M. C.-B. Williams et reçu par M. Donisthorpe.

Camponotus (Myrmotarsus) nigricans Rog. r. enganensis $\mathbf{n}$. st. ㅇ. Longueur: $18^{\mathrm{mm}}$. Tête, sans les mandibules, longue et large de $5^{\mathrm{mm}}$. Ailes teintes d'un jaune brunàtre. La tète, en forme de trapèze, a les còtés droit et-le bord postérieur convexe au milieu et des 2 còtés, avec une concavité au tiers latéral. Le scape, placé obliquement, dépasse à peine l'occiput. Tête mate, densément réticulée-ponctuée, avec une abondante ponctuation espacée. Le front, l'épistome, les lobes occipitaux et les joues sont luisants, seulement réticulés ou chagrinés. Le scape est distinctement déprimé. Mésonotum, scutellum et abdomen lisses et luisants. Epinotum et còtés du thorax réticulés et subopaques. La tète est bien plus large que le thorax. Noir; tarses et funicule bruns.

Ile d'Engano près Sumatra, reçu du Musée de Genève. Cette race diffère du type par sa taille plus grande (surtout la tète) et par la sculpture du vertex.

Camponotus (Myrmotrema) foraminosus For. s. str.

$\Varangle$ o St-Gabriel, Congo (KoHL), pris nombre de fois dans des plantes myrmécophiles, une fois dans une branche sèche de Cacao, une autre fois dans la Randia myrmecophila v. glabra de Wilde. 
q (encore inédite). Longueur: $10^{\mathrm{mm}}-11^{\mathrm{mm}}, 5$. Tout à fait semblable à l'ouvrière, en particulier pour la sculpture, la pubescence et la pilosité. Mésonotum avec deux arêtes parallèles, mat, ainsi que le scutellum. Les ailes sont colorées de brun roussâtre. Tête plus large que le thorax.

Camponotus (Orthonotomyrmex) chrysurus Gerst. r. acutisquamis Mayr.

$\sigma^{x}$ et $ᄋ$. $\sigma^{x}$ (encore inédit). Longueur : $11^{\mathrm{mm}}, 8$. Ailes d'un jaune brunâtre vers le bord antérieur. Mandibules avec une seule dent et le bord terminal tranchant. Epistome avec un lobe trapézoïdal, sans carène. Tête $1^{1 / 2}$ fois plus longue que large. Pubescence un peu plus faible que chez la $ᄋ$. Noir, tarses, mandibules et funicule brunâtre. Congo (KoHL).

Camponotus (Orthonotomyrmex) Meinerti For.

$\Varangle$ ․ Reçu aussi par M. Wasmann; les ouvrières font un peu passage à la r. Reginae For.

Camponotus (Orthonotomyrmex) Mayri For. r. Ledieui n. st.

ఢ. Longueur: $10^{\mathrm{mm}}$. Plus grand et surtout plus allongé que le type. Tête d' $1 / 5$ plus longue que large, avec le bord postérieur fortement convexe, pas plus large derrière les yeux qu'au $6^{\text {me }}$ antérieur. Diffère en outre du type par ses scapes fortement dilatés à leur moitié postérieure, par sa pilosité jaunâtre fine, bien plus abondante sur tout le corps, oblique sur les membres (longue et dressée sous les cuisses, L'épinotum et le nœud sont en outre grossièrement réticulés et de toute autre forme. L'épinotum n'est pas bordé ; sa face basale est très convexe et se termine derrière, après être redescendue, par une courte face déclive, concave et lisse, sans former d'angle avec elle. Le noud est aussi épais que sa hauteur, convexe en dessus, très peu plus large que long, avec ses faces antérieure et postérieure subverticales (un peu convexes). Cette race diffère de la r. Sankisianus For., surtout par sa sculpture, puis par la forme de l'épinotum et par sa forte pilosité. Le reste est comme chez le type, en particulier la fine sculpture mate, réticulée-ponctuée. 
Shilowana, Transvaal, récolté par M. Junod et reçu du Musée de Genève. Un seul exemplaire. Cette race mérite presque de constituer une espèce.

\section{Camponotus (Orthonotomyrmex) Maynei n. sp.}

$\Varangle$. Longueur: $5^{\mathrm{mm}}, 3-7^{\mathrm{mm}}$. $\Varangle$ major. Mandibules subopaques, finement réticulées et densément ponctuées : en outre de grosses fossettes, carieuses au fond. Tête d'un sixième plus longue que large, rétrécie et imprimée en travers à son sixième antérieur, puis rectangulaire derrière, avec les bords latéraux à peine convexes et le bord postérieur un peu concave; occiput un peu déprimé derrière. Arêtes frontales longues et divergentes. Epistome assez plat, rectangulaire, sans carène, un peu plus long que large, comme chez les Myrmotrema. Yeux au quart postérieur, assez plats. Le scape atteint tout juste l'occiput. Promésonotum peu convexe, subbordé. Pronotum 1 1/2 fois plus large que long. Mésonotum fortement rétréci derrière. Pas d'échancrure mésoépinotale, mais la suture est fortement marquée. Face basale de l'épinotum carrée, plate, obtusément bordée, légèrement plus longue que large. Face déclive, un peu plus longue que la face basale, plane comme elle, subverticale (faiblement oblique), formant avec la face basale un bord obtus et un angle de peutètre 95 degrés. Ecaille biconvexe, à bord tranchant, médiocrement épaisse. Cuisses légèrement renflées au milieu et un peu déprimées.

Entièrement subopaque, réticulé-ponctué, avec le thorax mat. La tête est en outre couverte d'abondantes fossettes carieuses au fond, moins denses sur l'occiput que sur l'épistome et les joues, tout à fait identiques à celles des Myrmotrema. Pilosité dressée assez courte et diluée, d'un blanc jaunâtre, assez épaisse et tronquée à l'extrémité, nulle sur les membres. Une rangée de soies blanches sur l'écaille et 3 ou 4 soies au bout des cuisses et des scapes. Pubescence presque nulle. Noir. Antennes et tarses roux, le reste des pattes et l'extrémité du funicule bruns.

$\Varangle$ minor. Tête en trapèze, bien plus large derrière que devant, un peu plus longue que large, avec les còtés droits et le

Rev. Suisse de Zool. T. 24. 1916. 
bord postérieur un peu convexe. Mandibules sculptées comme chez l'ouvrière major. Epistome caréné, de forme ordinaire, en trapèze élargi devant. Les yeux, très postérieurs, sont grands, distants du bord postérieur de la tête seulement du tiers de leur longueur. Le scape dépasse le bord postérieur de plus du quart, presque du tiers de sa longueur. Tout le reste, y compris la face basale de l'épinotum, identique à l'ouvrière major, mais la tête est entièrement mate et n'a aucune grosse fossette carieuse.

†. Longueur: $8^{\mathrm{mm}}, 9$. Tète plus longue que large, à còtés et bord postérieur presque droils, distinctement plus large derrière que devant, avec des angles postérieurs marqués, à courbe brusque. La tête est à peine plus large que le thorax. Face basale de l'épinotum, vue de profil, fortement convexe, séparée du postscutellum par une incisure profonde; elle est plus courte que la haute face déclive. Du reste comme l'ouvrière major, avec les mêmes fossettes sur la tête. Ailes presque hyalines, très faiblement teintées de jaunâtre, avec les nervures et la tache pâles.

Congo (R. Mayné) Musée de Bruxelles; reçu de M. SchouTEDEN.

Camponotus (Myrmamblys) Ferreri For. r. akka n. st.

$\zeta$ minor. Longueur: $5^{\mathrm{mm}}, 5$. Tête 2 fois plus longue que large, faiblement rétrécie d'avant en arrière, où son bord terminal est en même temps le bord articulaire. Epistome à bord antérieur laiblement convexe, en forme de trapèze élargi en avant, fort convexe, subcaréné au milieu. Les yeux sont au milieu. Le scape dépasse le bord postérieur des $\% / 5$ de sa longueur. Thorax très étroit (surtout le long épinotum), faiblement et également convexe d'un bout à l'autre. Ecaille conique, épaisse à la base, subacuminée au sommet. Pattes longues et minces.

Luisant, faiblement chagriné. Pilosité dressée, très éparse sur le corps, nulle sur les membres; pubescence très courte et dilıée. Tête et abdomen bruns; tout le reste d'un roux jaunâtre pâle un peu brunâtre. 
१. Longueur: $9^{\mathrm{mm}}$. Diffère du type (basé sur la $q$ ) par sa pilosité plus éparse, par sa sculpture plus faible devant et par ses mandibules a bord externe courbé. La taille est aussi plus grande, le bord postérieur de la tète plutòt un peu concave au milieu. Epistome subcaréné comme chez l'ouvrière minor. Les yeux sont plus petits, n'occupant guère que le quart des còtés. Le scape est plus long dépassant la tête de plus de 3 fois son épaisseur. La face basale de l'épinotum est bien convexe, mais ne remonte pas distinctement en arrière. Les tibias sont comme chez le type, sans petits piquants. Tout le corps est luisant, faiblement chagriné. Couleur comme chez l'ouvrière minor, mais l'épistome et les joues sont d'un roux jaunâtre bien moins distinct du brun foncé de la tète que chez le type.

Congo (KонL).

Polyrhachis (Hagiomyrma) ammonoeides Rog. v. Crawleyi n. var.

ఢ. Longueur: $5^{\mathrm{mm}}, 5-6^{\mathrm{mm}}, 5$. Plus petite que le type. La tête est plus allongée, $1 \frac{1}{4}$ fois plus longue que large, son bord postérieur est bien plus étroit et bien plus convexe que chez le type; la pelisse de l'abdomen est un peu moins mordorée, plus simplement dorée. Du reste identique.

Australie du Nord, reçue de M. Crawley.

Polyrhachis (Myrma) laboriosa Sm.

భ o St-Gabriel, Congo (KoнL). Dans un nid composé de feuilles, reliées entre elles par un làche tissus de fils entremêlés de matériaux végétaux.

Polyrhachis (Myrma) militaris F. r. cupreopubescens For. v. nkomoensis $\mathrm{n}$. var.

ఢ. Longueur : $10^{\mathrm{mm}}, 5-12^{\mathrm{mm}}$. L'épinotum a des épines verticales et fortement recourbées en avant (2 fois plus longues que l'épaisseur de leur base) au lieu de dents, rappelant ainsi la v. epinotalis For., dont elle diffère du reste par sa tête non rétrécie derrière et par sa sculpture qui est comme chez le type de la race. La sculpture est moins dorée que chez le type. Dans ma description de l'epinotalis, j'ai négligé de rendre 
attentif à sa tête fortement rétrécie derrière les yeux, jusqu'à un bord postérieur presque confondu avec le bord articulaire. Les còtés rétrécis sont néanmoins convexes et les yeux sont très convexes, presque hémisphériques. La stature est aussi plus grêle que chez le type de la cupreopubescens, de sorte que je crois devoir faire de l'epinotalis une race séparée.

․ Longueur: $12^{\mathrm{mm}}, 5-13^{\mathrm{mm}}$. Tout à fait semblable au type de la race; ailes brunâtres comme chez le type de l'espèce ; thorax un peu plus large que la tête. L'épinotum n'a que des dents pointues, un peu plus longues que larges.

$\sigma^{x}$. Longueur: $7^{\mathrm{mm}}-7^{\mathrm{mm}}, 5$. Ailes un peu plus claires que chez la $q$. Subopaque ou mate. Pelisse plus faible que chez la $\{$. Tête $1 \frac{1}{3}$ fois plus longue que large, avec un bord postérieur convexe assez net.

Congo (КонL); prise avec le Camponotus (Myrmoturba) maculatus Brutus, faisant la chasse aux Termites. Reçue aussi par M. Wasmann.

Polyrhachis (Myrma) concava André.

¡ o Congo (КонL).

+ (encore inédite). Longueur: $7^{\mathrm{mm}}, 5$. Tête comme chez l'ouvrière, plus étroite que le thorax; ce dernier n'a de concavité qu'entre les deux petites dents obtuses de l'épinotum, dont la face basale, 2 fois plus large que longue, fortement bordée, est convexe en avant et a les bords latéraux convexes. Le scutellum est subcaréné au milieu. Les ailes manquent. Du reste comme l'ouvrière.

Polyrhachis (Myrma) Alluaudi Em. v. anteplana n. var.

ఢ. Longueur: $6^{\mathrm{mm}}, 3$. Diffère du type de l'espèce, d'après la description, par son épinotum et ses dents plus longues, ainsi que par son pronotum qui est presque plat. La fissure transversale mésoépinotale est verticale, très étroite et profonde. Les dents de l'épinotum sont triangulaires, un peu recourbées en avant; les épines du pronotum sont moins de 2 fois plus longues que la largeur de leur base.

Congo (КонL). 
Polyrhachis (Myrma) Otleti n. sp.

భ. Longueur: $6^{\mathrm{mm}}, 7$. Appartient au groupe de la P. Revoili André et lanuginosa Sant., dont elle diffère par son pronotum bordé. Mandibules subopaques, très finement réticulées, avec d'abondants gros points enfoncés, armées de 5 dents. Tête à peine d' $1 / 5$ plus longue que large, à bord postérieur convexe et à còtés peu convexes, un peu plus étroite devant que derrière. L'épistome est caréné, peu convexe, fortement avancé en avant en lobe trapéziforme. Le bord antérieur du lobe est court, droit, à peine subdenté à l'extrémité. Arêtes frontales longues, sinueuses, divergentes. Yeux fort convexes, presque semicirculaires. Le scape dépasse le bord postérieur de presque la moitié de sa longueur. Thorax bordé, à dos assez convexe en tout sens. Les bords du mésonotum et de l'épinotum sont obtus, ceux du pronotum aigus, convexes, se prolongeant devant en deux épines, longues comme 1 1/2 fois la largeur de leur base. Pronotum $1 \frac{1}{1} / 2$ fois plus large que long; le mésonotum un peu moins large; les deux sutures distinctes. Face basale de l'épinotum rectangulaire, 1 1/4 fois plus large que longue, séparée de la face déclive par un rebord transversal très net, un peu interrompu au milieu. La face déclive est oblique, un peu plus courte que la basale, à peine subbordée, un peu concave de profil. Le pétiole a une dent en dessous. Son écaille épaisse et biconvexe est un peu plus haute qu'épaisse, armée de 4 épines, dont les supérieures, 2 fois plus longues que les latérales, sont courbées en arrière, à peine plus courtes que leur large intervalle; le bord supérieur est un peu convexe. Face antérieure de l'abdomen tronquée, un peu concave (faiblement); pattes plutòt épaisses, comme la stature de tout le corps qui est robuste.

Mate; abdomen à peine un peu subopaque. Fine sculpture foncière densément réticulée-ponctuée, aussi sur les membres. En outre, la tête et le thorax sont ridés en long, plutòt réticulés sur les còtés. Ces rides et réticulations sont denses, médiocrement grossières. Tout le corps et les membres abondamment recouverts d'une pilosité dressée, fine, pointue, jaunâtre, et 
assez longue, un peu moins abondante sur l'abdomen qui a par contre une pubescence assez diluée, jaunâtre, plus abondante que la tête et le thorax, de mème que les membres. Entièrement noire, avec les membres brun foncé; seconde moitié des funicules et extrémité des mandibules roussâtres.

q. Longueur : $8^{\mathrm{mm}}$. Toute semblable à l'ouvrière. Tète un peu plus étroite que le thorax. Le pronotum n'a que 2 fortes dents triangulaires. Epinotum, écaille et tout le reste comme chez l'ouvrière. Ailes brunes. Sur le scape on voit très distinctement deux sortes de poils, les uns très longs et les autres plus courts.

$\sigma^{x}$. Longueur: $6^{\mathrm{mm}}, 7$. Tête un peu plus large que longue. Epistome caréné, plus convexe que chez l'ouvrière, à bord antérieur convexe, sans lobe. Arêtes frontales très rapprochées devant et très divergentes derrière. Tête un peu plus étroite que le thorax. Ecaille sans épines, épaisse, arrondie au sommet. Articles médians du funicule seulement un peu plus longs qu'épais. Pilosité et pubescence plus faibles que chez l'ouvrière. Couleur identique à celle de la $q$, mais les funicules sont entièrement bruns.

St-Gabriel, Congo (KoHL). Nid dans la fente d'une écorce d'arbre qui, sur une longueur de $8 \mathrm{~cm}$. et une largeur de $2 \frac{1}{\mathbf{2}}$, ètait recouverte d'une toile filée, mêlée de matériaux végétaux.

\section{Polyrhachis (Myrma) decemdentata André.}

ㅇ Congo (KонL).

\section{Polyrhachis (Myrma) Spitteleri n. sp. (Fig. 6.)}

ఈ. Longueur: $4^{\mathrm{mm}}, 5$. Mandibules subopaques, très densément striées, avec des points épars et le bord externe assez convexe. Tête en trapèze arrondi, un peu plus longue que sa largeur postérieure (au quart postérieur, où elle est large comme $1 \frac{1}{1 / 2}$ fois le bord antérieur), avec le bord postérieur très convexe. Epistome caréné, biéchancré, convexe, mais sans lobe bien accentué au bord antérieur. Arêtes frontales, sinueuses, divergentes. Yeux médiocrement convexes, un peu en avant du tiers antérieur. Le 
scape dépasse l'occiput d'un fort tiers de sa longueur. Thorax assez convexe, surtout le promésonotum, nullement bordé. Pronotum 1 1/3 fois plus large que long, bien plus large devant que derrière et moins convexe que chez la monista, portant devant 2 épines un peu aplaties, tout à fait analogues à celles de la monista, mais d'un tiers plus courtes et dirigées subhorizontalement en avant et de còté. Suture promésonotale très distincte, mais nullement enfoncée, au contraire de la monista. Mésonotum 2 1/2 fois plus large que long. Au lieu d'échancrure, une incisure étroite entre le mésonotum et l'épinotum, mais de moitié moins profonde que chez la monista. Face basale de l'épinotum rectangulaire, $1 \frac{1}{3}$ fois plus large que longue et terminée par 2 longues épines

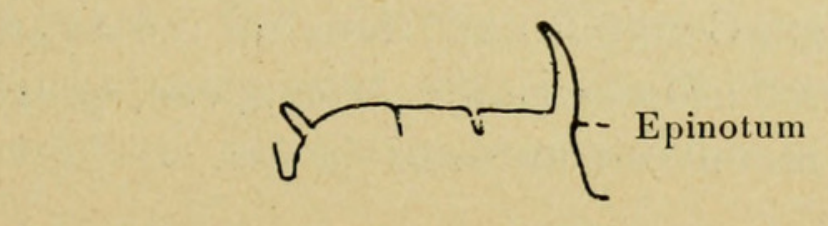

FIg. 6.

Polyrhachis (Myrma) Spitteleri For. $\Varangle \quad$ (Emma Forel, del.)

Profil du thorax.

divergentes, subverticales, un peu recourbées en avant, plus longues que la face basale qui est assez horizontale. Face déclive subverticale, à peine plus courte que la basale avec laquelle elle forme un angle presque droit, à peine obtus. Ecaille épaisse, biconvexe, mais bien plus haute qu'épaisse, surmontée de 4 épines équidistantes, toutes courbées en arrière, comme chez. la monista, les supérieures plus longues que leur intervalle, mais plus courtes et moins fortes que les latérales. Abdomen tronqué et un peu concave devant.

Tout le corps mat, finement et densément réticulé-ponctué ; les membres plutôt subopaques avec la même sculpture. La tête et le thorax sont en outre densément striés-ridés en long (pas très grossièrement). Sur l'abdomen les réticulations font transition à des rides longitudinales plutòt fines. Le corps, surtout le thorax, et aussi en partie les tibias sont couverts de la même pilosité épaisse, presque tronquée, rousse et hérissée 
qu'on voit chez la monista. Cuisses et antennes sans poils dressés. Pubescence jaunâtre, très courte et adjacente, surtout visible sur les membres, où elle est diluée, presque nulle ailleurs. Noire. Tibias, funicules, tarses et extrémité des mandibules brunâtres.

Congo (KонL) ( $\left.\mathrm{N}^{\circ} 5\right)$. Cette curieuse espèce (un seul exemplaire) est très proche parente de la monista Sant., dont elle diffère essentiellement par le manque complet d'incisure promésonotale ainsi que par son épinotum cubique. Le corps est bien moins strié et plus ridé, mat et non pas luisant. Le mésonotum est ridé en long au lieu d'être strié en travers, etc.

\section{Polyrhachis (Myrma) monista Sant.}

¡ Kilongalonga, Congo (KонL), dans un nid de carton qui se trouvait dans une feuille enroulée. Je suppose, qu'ici comme ailleurs, le carton recouvrait un tissu de fils.

\section{Polyrhachis (Myrma) Revoili André.}

¡ $ᄋ \sigma^{x}$ Congo (KoнL); pris ensemble. La longueur des épines varie chez l'ouvrière. Chez les exemplaires de M. Конц les épines inférieures sont longues comme les ${ }^{2 / 3}$ des supérieures, chez d'autres seulement comme la moitié. L'abdomen est subopaque, parfois assez luisant. Le bord postérieur de la tête est peu convexe, presque 2 fois plus large que son bord antérieur extrême. La tète est densément ridée-striée en long, les épines du pronotum 2 fois plus longues que la largeur de leur base. Le thorax est plutòt réticulé avec direction longitudinale, mais un peu ridé sur le pronotum, dont les épines sont plus larges à la base chez les individus de M. Конц. Longueur : $5^{\mathrm{mm}}-6^{\mathrm{mm}}, 5$.

La femelle, seule décrite par André, a les ailes brunes. Celles de M. КонL sont longues de $6{ }^{m m} 7-7^{\mathrm{mm}}$. L'épinotum qui, chez l'ouvrière, a une dent pointue presque verticale et très nette, est, chez la $q$, soit complètement inerme, comme le dit André, soit parfois muni d'une petite dent obtuse. Suivant les individus, le mésonotum est simplement réticulé ou il a en outre quelques rides longitudinales.

$\sigma^{x}$ (encore inédit). Longueur: $4^{\mathrm{mm}}, 5-5^{\mathrm{mm}}$. Ailes d'un brun à 
peine plus pâle que chez la †. Mandibules armées de 4 dents. Tête avec un large bord postérieur convexe, presque 2 fois plus large que le bord antérieur. Finement réticulé et subopaque sans sculpture grossière. Ecaille très épaisse et basse, sans dents. Pilosité dressée, éparse partout, très courte et oblique sur les tibias et les scapes.

Je ne puis trouver de différences entre Revoili et la description de natalensis Sant. Weissi Sant., ne me paraît être qu'une variété plus striée sur le thorax, comme l'indique Santschi. M. SAntschi a eu la bonté de m'indiquer encore par lettre quelques autres différences.

\section{Polyrhachis (Myrma) Revoili André v. Donisthorpei n. v.}

$\underset{+}{\dddot{\sigma}}$ Longueur: $5^{\mathrm{mm}}, 2-5^{\mathrm{mm}}, 6$. Epines pronotales bien plus longues que chez le type, mais pilosité identique. Forme de la tète et sculpture, par contre, comme chez la variété conduensis For.

North Rhodesia, reçue par M. H. Donisthorpe.

\section{Polyrhachis (Myrma) Revoili André r. Volkarti n. st.}

․ Longueur : $6^{\mathrm{mm}}, 5$. Beaucoup plus grêle que le type de l'espèce. La tête est beaucoup plus étroite et plus allongée, d'un quart plus longue que large, avec bord postérieur un peu rétréci derrière les yeux, a peine plus large que le bord antérieur. C'est au bord postérieur des yeux que la tête est le plus large. L'épistome caréné à un lobe antérieur bien plus long que chez le type. Le scape dépasse le bord postérieur de plus de la moitié de sa longueur (du moins chez le type). Thorax bien plus large que la tête. Pronotum bidenté. L'épinotum a deux angles à peine dentiformes; il est du reste comme chez le type. Ecaille plus étroite et plus haute que chez le type; ses épines supérieures, fortement recourbées en arrière à l'extrémité, ne divergent que faiblement. Les épines latérales sont très courtes, seulement un peu plus longues que la largeur de leur base. Tout l'Insecte est mat, densément réticulé-ponctué ; les cuisses seules sont subopaques. La tête est longitudinalement ridée; de même les còtés de l'abdomen. Le mésonotum a quelques 
faibles rides longitudinales. La pilosité dressée est notablement moins abondante que chez le type, surtout sur l'abdomen où elle est rare; aussi un peu éparse sur les membres. La pubescence est, par contre, au moins aussi abondante que chez le type, mais un peu plus courte. Noire, Mandibules et membres bruns ; moitié terminale du funicule roussâtre.

Congo (КонL). Un seul individu.

L'étude des formes précédentes montre les transitions du Sous-Genre Myrma Bill. au Sous-Genre Cyrtomyrma For. par les formes Otleti, Spitteleri, Monista et Revoili. On peut douter si l'on doit rattacher ces trois dernières formes au Cyrtomyrma ou au Myrma. Par contre, pour les deux suivantes, surtout pour l'Alexisi, je crois que le doute n'est guère possible.

\section{Polyrhachis (Cyrtomyrma) Kohli n. sp.}

భ. Longueur : $4^{\mathrm{mm}}, 5--5^{\mathrm{mm}}, 5$. Mandibules subopaques, densément ponctuées et striées vers la base, armées de 4 dents, à bord externe peu convexe. Tout le corps très étroit et grêle. Tête $1 \frac{1}{2}$ foị plus longue que large, sans bord postérieur autre que le bord articulaire.Derrière les yeux, ses côtés constituent une forte convexité, à peu près semicirculaire; devant les yeux, ils sont presque droits, convergeant un peu en avant. Situés au milieu, les yeux sont fort convexes, presque semicirculaires, occupant presque le quart des còtés. Epistome sans carène, avancé devant en lobe arqué, assez convexe de profil. La tête est assez épaisse de dessous en dessus, bordée en dessous d'une arête longitudinale. Le front est peu convexe devant, mais fortement courbé au milieu des arêtes frontales qui, jusque-là, sont distinctement convergentes et assez relevées; puis elles divergent modérément en S. Le scape dépasse le bord articulaire de la tête des $\% / 5$ de sa longueur. Le thorax est bien 3 fois plus long que large. Le pronotum, un peu plus long que large, a deux dents triangulaires assez obtuses, un peu plus longues que l'épaisseur de leur base. Mésonotum d'un quart plus large que long. Face basale de l'épinotum d'un quart plus longue que large, bordée derrière d'une arête vive assez élevée et interrompue au milieu par une échancrure. De côté, cette arête constitue un 
angle dentiforme, à partir duquel elle se continue en avant, bordant de còté les premiers $2 / 5$ de la face basale. Le reste du dos du thorax n'est pas bordé, à part la première moitié du pronotum, devant et de còté, bordée par la continuation de sa dent. Face déclive de l'épinotum un peu plus courte que la basale, ayant vers le bas deux stigmates proéminant en dents obtuses et une petite carène médiane vers le haut. Ecaille très épaisse, biconvexe, presque aussi épaisse que sa hauteur, surmontée en haut de deux épines droites, subverticales (peu divergentes), aussi longues que leur intervalle. De còté, elle a en outre 2 dents latérales pointues, plus longues que larges et rapprochées des épines. Abdomen un peu concave devant, vers le bas. Pattes longues et grêles.

Subopaque. La sculpture consiste partout en réticulations plutôt fines, entremêlées sur la tête et le thorax de rides longitudinales plus ou moins distinctes et pas très grossières. Pilosité dressée, fine, jaunâtre, plutôt longue, abondante sur la tête, le thorax, les tibias et les scapes, moins abondante sur l'abdomen et les cuisses. Pubescence longue et très distincte, également répartie sur tout le corps et les membres, jaunâtre, mais ne formant nulle part un duvet et ne cachant nullement la sculpture. Noire; membres d'un brun foncé; une grande partie des tarses et des tibias, extrémité des mandibules, et les \%/3 terminaux des funicules roussâtres.

ๆ. Longueur: $6^{\mathrm{mm}}, 5$. Identique à l'ouvrière, mais le pronotum n'a que 2 petites dents obtuses. Thorax un peu plus large que la tête et presque entièrement mat. Les ailes manquent. Les épines supérieures de l'écaille sont à peine plus longues que les $2 / 5$ de leur intervalle; les dents latérales pas plus longues que larges. Tout le reste comme chez l'ouvrière.

Congo (КонL). Cette espèce est remarquable par sa taille étroite et grêle.

Polyrhachis (Cyrtomyrma) Alexisi n. sp. (Fig. 7.)

ఢ. Longueur : $3^{\mathrm{mm}}, 5-4^{\mathrm{mm}}$. Mandibules luisantes, assez lisses, faiblement ponctuées, armées de 5 dents. Tête trapéziforme, un peu plus longue que sa largeur vers les yeux, à còtés très peu con- 
vexes. Le bord postérieur, faiblement convexe, est large au moins $1 \frac{1}{2}$ fois comme le bord antérieur. Epistome convexe, sans carène, avec un lobe antérieur trapéziforme, bidenté devant, droit entre les dents. Arêtes frontales très rapprochées entre les articulations des antennes, divergentes devant et derrière où elles sont sigmoïdes. Yeux au quart postérieur. Le scape dépasse l'occiput d'au moins les $2 / 5$ de sa longueur. Thorax 1 1/2 fois plus long que large. Pronotum 2 fois plus large que long, bordé devant et de còté jusqu'à sa moitié, formant à ses angles antérieurs une dent très large, subhorizontale, assez pointue, mais à base si large qu'elle ne la dépasse que de peu, tout en constituant l'angle qui-sépare les còtés du devant. Mésonotum

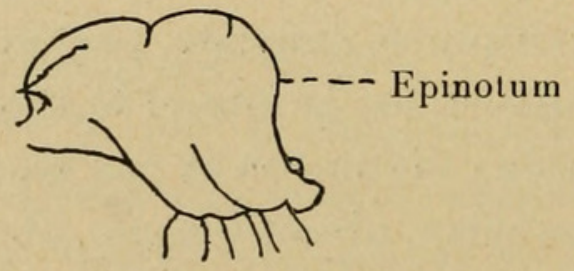

Fig. 7.

Polyrhachis (Cyrtomyrma) Alexisi For. $\Varangle$ (Emma Forel, del.) Profil du thorax.

presque 3 fois plus large que long. Suture mésoépinotale peu distincte. Tout le thorax est fortement convexe en tout sens. Mais l'épinotum, extrêmement haut, est aussi extrêmement court, formant derrière, d'abord une forte convexité, dans un sens presque vertical, puis, plus bas, une concavité allant jusqu'à l'articulation. On peut à peine distinguer une apparence d'angle entre ce qui serait une face basale convexe et une face déclive concave. De droite à gauche, l'épinotum est, de même, fortement convexe, sans trace de bord, de dents ni d'épines. Ecaille biconvexe, assez épaisse, surmontée de deux épines droites, un peu plus longues que la moitié de leur intervalle, et de deux dents latérales pointues, un peu plus longues que larges. Abdomen très convexe.

Subopaque. Tête densément striée en long, plutòt réticulée sur les côtés, avec l'épistome assez finement et irrégulièrement réticulé. Tout le reste du corps est densément réticulé ou réti- 
culé-ponctué, avec quelques rides longitudinales assez indistinctes sur le thorax. Pilosité dressée nulle, saufaux deux extrémités du corps, qui ont quelques poils. Pubescence très courte et très diluée sur le corps, un peu plus distincte sur les membres, jaunâtre. Noire, membres brun foncé ; funicule, sauf le premier article, et extrémité des mandibules, d'un roux jaunâtre.

ㅇ. Longueur: $5^{\mathrm{mm}}, 5$. Tête beaucoup plus longue que chez l'ouvrière, d'un bon quart plus longue que large, en trapèze peu marqué, seulement un peu plus étroite devant que derrière; le bord postérieur est peu convexe. Thorax plus large que la tête, court, haut. L'épinotum a une face basale très convexe, mais assez marquée, passant par une courbe à une face déclive subverticale et deux fois plus haute qu'elle. Abdomen tronqué et un peu concave devant (chez l'ouvrière aussi). L'épinotum a des rides transversales assez distinctes, tandis que le mésonotum et le scutellum ont la même sculpture que chez l'ouvrière. Sur la partie postérieure de l'abdomen les réticulations tendent à se transformer en ponctuation très dense. Les ailes manquent. Tout le reste comme chez l'ouvrière.

Congo (Kонl). Cette curieuse espèce ressemble à la P. Cyrtomyrma) laevissima Sm., mais s'en distingue non seulement par sa petite taille et par sa sculpture, mais encore par la grande hauteur de son épinotum bossu. Je la dédie à la mémoire de mon grand-oncle Alexis Fonel qui guida mes premiers pas dans l'entomologie dès 1859.

Nous devons à M. Hermann KoHL, outre de nouvelles formes de Fourmis très intéressantes, entre autres celles des plantes myrmécophiles, de fort importantes données biologiques qui viennent éclaircir les mœurs de beaucoup de formes.

\section{APPENDICE.}

L'interdiction récente de répéter le même nom dans le mème genre pour les variétés me contraint à renommer les formes suivantes :

Camponotus (Myrmoturba) Gallienii n. sp. de Madagascar (= concolor For. 1891 nec. Alii v. concolor 1890). 
Camponotus (Myrmoturba) oblongus Sm. v. binominata n. var. (= v. opaca For. 1907, nec. Emery 1899).

Camponotus (Myrmoturba) maculatus F. r. carolinus $\mathbf{n}$. st. (=luteolus Em. 1905 nec. r. hova For. v. luteola For. 1897).

Camponotus (Orthonotomyrmex) lateralis Ol. r. spissinodis n. st. (déjà renommé) (= crassinodis For. 1894, nec. C. maculatus F. r. mitis Sm. v. crassinodis For. 1892).

Dolichoderus Taschenbergi Mayr. v. wheeleriella n. var. (=v. gagates Wh. 1905, nec. sp. gagates Em. 1890).

De plus je me permets de donner le nom d'Esdras n. var. à une petite variété du Camponotus (Myrmobrachys) planatus Rag. longue de $4-4^{\mathrm{mm}}, 3$, provenant d'Orizaba, au Mexique, variété qui se distingue par sa couleur tachetée de roussâtre et de brun sur la tète, le thorax et l'abdomen. Je l'avais appelée variegata in litt., nom déjà employé par Sm. en 1858.

Dernièrement M. Enery (Académie des Sciences de Bologne, 21 mars 1915, page 6) a institué un nouveau sous-genre qu'il nomme Attomyrma du genre Aphaenogaster, avec subterranea comme type. La seule différence appréciable consiste dans le thorax étroit des femelles chez les Aphaenogaster s. str. (type testaceo-pilosa Luc.). M. Emery supposait alors que les Aphaenogaster sens. str. n'avaient pas d'ailes chez la $q$ ou simplement des rudiments, ce qui est une erreur, car je possédais depuis longtemps des $q$ ailées de testaceo-pilosa et plus tard M. EMery en a élevé lui-mème. M. Emery place de plus les Aphaenogaster longiceps et Sagei For. dans ses Attomyrma, lors même que ce dernier n'a qu'une seule cellule cubitale aux ailes. Le fait d'avoir le thorax plus ou moins large variant suivant les races d'une même espèce, même parfois suivant les individus (M. Emery cite lui-mème la v. Tyrrhena du Messor barbarus structor), je ne puis admettre qu'on fonde un sous-genre sur pareils caractères et je me refuse à adopter le sous-genre Attomyrma Em., qui contient des formes hétérogènes. Je laisse le Sagei dans le sous-genre Deromyrma For.

M. Emery m'écrit qu'il considère mon Atopula Jacobsoni (Fauna simalurensis 1915, page 25) comme un Leptothorax. Je suis d'accord (voir ibidem page 27), mais dans le cas il faudra 
aussi faire de l'A. ceylonica Em., avec la var. Taylori For., des Leptothorax et non des Atopula.

M. Emery a fondé une r. aegyptiacus du C. (Myrmoturba) maculatus F., qui n'est à mon avis qu'une simple variété à tibias jaunes (Emery, Bull. Soc. Ent. France, 10 février 1915, p. 79). En effet: $1^{\circ}$ M. Emery ne cite pas les caractères de la couleur du corps du maculatus indiqués par Latreille. $2^{\circ} \mathrm{Je}$ possède moi-même des maculatus typiques à tibias brun-noir provenant de l'Afrique équatoriale $\left(\stackrel{q}{q} \sigma^{x}\right)$ qui ne sont pas le melanocnemis Sant. $3^{\circ}$ M. Emery n'indique pas les caractères les plus importants du melanocuemis. Ce dernier n'est en tout cas pas synonyme de la var. aegyptiaca Em. qui ne me paraît différer du type que par la couleur des tibias.

M. Emery pense que mon sous-genre Caulomyrma (de Leptothorax) est synonyme du genre Nesomyrmex Wh. Ce dernier n'est fondé que sur une seule $\subsetneq$ et se distingue, outre les antennes de 11 articles, par sa tête bordée derrière. Ne connaissant pas le type de Nesomyrmex, je maintiens, provisoirement du moins, et jusqu'à plus ample information le sous-genre Caulomyrma. Quant à la suppression, ou plutòt à la transformation en sous-genre du genre Dicroaspis Em., je pense aussi qu'il vaudrait peut-être mieux attendre de plus amples connaissances.

Dans le Bulletin de l'American Museum of Natural History, 1915, p. 395, M. Wheeler croit devoir identifier ma Solenopsis pylades avec la saevissima Sm., prétendant que la description de Sмiтн s'applique parfaitement à la Pylades jaune. Il en fait de plus une simple race de geminata, disant que Xyloni Mac Cook et maniosa Wh. sont intermédiaires. Je ne puis absolument pas admettre la synonymie de M. Wheeler, car : 1) je possède des geminata et des pylades de toutes les parties de l'Amérique tropicale à l'est des Andes; 2) Je conteste que la description de Sмiтн permette de savoir à laquelle des deux espèces appartient sa saevissima. Il ne dit pas un mot de la structure des mandibules et parle même distinctement d'un " worker minor » qu'il décrit séparément de la grande $\zeta$, ce qui va bien mieux à la geminata qu'à la Pylades. Je maintiens donc le nom de 
Pylades. Reste la question des intermédiaires que je n'ai jamais vus ni du Brésil, ni de Colombie, ni de l'Argentine où la Pylades seule paraît exister. La différence entre les ouvrières major et les $q$ de Pylades et de geminata est si grande que jusqu'à nouvel ordre je crois devoir maintenir la différence spécifique, malgré les formes plus ou moins intermédiaires des Etats-Unis. A bien des égards les $S$. aurea Wh. et Gayi Spin. ne sont certes pas plus différentes de geminata que la Pylades.

Enfin je propose le nom de Myrmoplatys n. subgenus pour le groupe asiatique des Myrmomalis, qui décidément diffère trop du groupe néotropique pour y demeurer. Type: Korthalsiae Em. Dans les Myrmoplatys c'est surtout chez la of et chez l'ouvrière, seulement devant, que la tête est déprimée. La chitine est en général lisse.

MM. Morrice et Durrant (Trans. Ent. Soc., London 1914 , page 421 [1915]) ont institué en remplacement du Genre Lasius F. (1804) qu'ils disent tombé par synonymie de Lasius Jurine (1801, Apide), un nouveau Genre Donisthorpea. Mais ces auteurs ne tiennent aucun compte des sous-genres Acanthomyops Mayr. Dendrolasius Ruszky et Chthonolasius Ruszky, dont le dernier est à mon avis synonyme de Lasius s. str. et ne pouvait être maintenu. Donc, suivant mon opinion, la synonymie doit être la suivante, si Morrice et Durrant ont raison:

Genre Acanthomyops Mayr. (1862)

$=$ Lasius F. 1804 (non Jurine 1801)

$=$ Donisthorpea Morrice et Durrant;

Type : claviger Roger,

Subgen. Chthonolasius Ruszky, type : niger L. (flavus ex. Ruszky).

Sugen. Dendrolasius Ruszky, type : fuliginosus Latr.

P. S. M. Emery m'écrit qu'à son avis il vaudrait mieux prendre pour Lasius le nom nouvellement déterré par Wheeler de Formicina Shuck., nom en partie basé sur le Lasius flavus. Je n'ai rien à y opposer, pourvu qu'on en finisse une bonne fois avec ces déménagements perpétuels des anciens noms. 


\section{$2 \mathrm{BHL}$ Biodiversity Heritage Library}

Forel, Auguste. 1916. "Fourmis du Congo et d'autres provenances récoltées par MM. Hermann, Kohl, Luja, Mayné, etc." Revue suisse de zoologie 24, 397-460. https://doi.org/10.5962/bhl.part.4645.

View This Item Online: $\underline{\text { https://www.biodiversitylibrary.org/item/40346 }}$

DOI: https://doi.org/10.5962/bhl.part.4645

Permalink: https://www.biodiversitylibrary.org/partpdf/4645

\section{Holding Institution}

MBLWHOI Library

Sponsored by

MBLWHOI Library

\section{Copyright \& Reuse}

Copyright Status: NOT_IN_COPYRIGHT

This document was created from content at the Biodiversity Heritage Library, the world's largest open access digital library for biodiversity literature and archives. Visit BHL at https://www.biodiversitylibrary.org. 